\title{
Enantioselective Total Synthesis of Cerorubenic Acid-III via Type II
}

\section{[5+2] Cycloaddition Reaction}

Xin Liu ${ }^{\dagger \#}$, Junyang Liu ${ }^{\dagger, * \#, ~ J i a n l e i ~} W u^{\dagger}$ and Chuang-Chuang $\mathrm{Li}^{\dagger *}$

†Shenzhen Key Laboratory of Small Molecule Drug Discovery and Synthesis, Shenzhen Grubbs

Institute, Department of Chemistry, Guangdong Provincial Key Laboratory of Catalytic

Chemistry, Southern University of Science and Technology, Shenzhen 518055, China

$\ddagger$ Academy for Advanced Interdisciplinary Studies, Southern University of Science and Technology,

Shenzhen 518055, China

ccli@s,sustech.edu.cn

\# These authors contributed equally to this work.

\section{Supplementary Materials}

Table of Contents

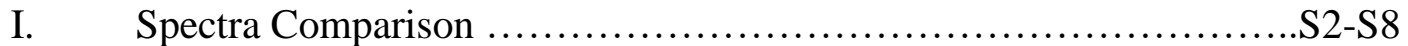

II. X-ray Crystallographic Data .......................................... 911

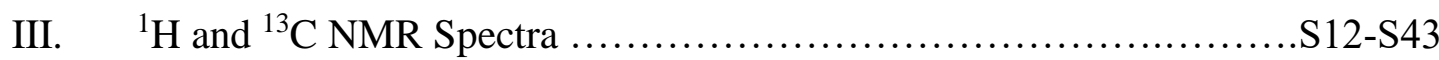




\section{Spectra Comparison}

Table S1. ${ }^{1} \mathrm{H}(500 \mathrm{MHz})$ and ${ }^{13} \mathrm{C}(126 \mathrm{MHz})$ NMR data of cerorubenic acid-III (1) in $\mathrm{CD}_{2} \mathrm{Cl}_{2}(\delta \text { in ppm, } J \text { in } \mathrm{Hz})^{a}$

\begin{tabular}{|c|c|c|}
\hline position & $\delta_{\mathrm{H}}$ & $\delta_{\mathrm{C}}$ \\
\hline 1 & $1.84(\mathrm{~m}, 1 \mathrm{H})$ & 36.5 \\
\hline 2 & $0.68(\mathrm{t}, J=9.9 \mathrm{~Hz}, 1 \mathrm{H})$ & 31.2 \\
\hline 3 & - & 21.0 \\
\hline 4 & $1.63(\mathrm{~m}, 1 \mathrm{H}), 1.35(\mathrm{~m}, 1 \mathrm{H})$ & 29.1 \\
\hline 5 & $2.00(\mathrm{~m}, 1 \mathrm{H}), 1.79(\mathrm{~m}, 1 \mathrm{H})$ & 24.3 \\
\hline 6 & $5.69-5.62(\mathrm{~m}, 1 \mathrm{H})$ & 124.6 \\
\hline 7 & - & 140.9 \\
\hline 8 & $2.49-2.42(\mathrm{~m}, 1 \mathrm{H}), 2.20(\mathrm{~m}, 1 \mathrm{H})$ & 33.0 \\
\hline 9 & $2.20(\mathrm{~m}, 1 \mathrm{H}), 1.50(\mathrm{~m}, 1 \mathrm{H})$ & 28.9 \\
\hline 10 & $2.32(\mathrm{~m}, 1 \mathrm{H})$ & 40.8 \\
\hline 11 & - & 152.3 \\
\hline 12 & $2.20(\mathrm{~m}, 1 \mathrm{H}), 2.15(\mathrm{~m}, 1 \mathrm{H})$ & 32.9 \\
\hline 13 & $1.65(\mathrm{~m}, 1 \mathrm{H}), 1.57(\mathrm{~m}, 1 \mathrm{H})$ & 24.2 \\
\hline 14 & $1.20(\mathrm{~m}, 1 \mathrm{H})$ & 45.0 \\
\hline 15 & $1.70(\mathrm{~m}, 1 \mathrm{H})$ & 32.6 \\
\hline 16 & $1.56(\mathrm{~m}, 1 \mathrm{H}), 1.16(\mathrm{~m}, 1 \mathrm{H})$ & 32.8 \\
\hline 17 & $2.27(\mathrm{~m}, 1 \mathrm{H}), 2.12(\mathrm{~m}, 1 \mathrm{H})$ & 27.1 \\
\hline 18 & $6.90(\mathrm{t}, J=7.6 \mathrm{~Hz}, 1 \mathrm{H})$ & 146.1 \\
\hline 19 & - & 127.1 \\
\hline 20 & - & 173.2 \\
\hline 21 & $1.83(\mathrm{~s}, 3 \mathrm{H})$ & 12.3 \\
\hline 22 & $0.91(\mathrm{~d}, J=6.7 \mathrm{~Hz}, 3 \mathrm{H})$ & 18.2 \\
\hline 23 & $1.10(\mathrm{~s}, 3 \mathrm{H})$ & 27.5 \\
\hline 24 & $0.96(\mathrm{~d}, J=9.2 \mathrm{~Hz}, 1 \mathrm{H})$ & 25.0 \\
\hline 25 & $4.76(\mathrm{~d}, J=2.0 \mathrm{~Hz}, 1 \mathrm{H}), 4.70(\mathrm{~d}, J=2.1 \mathrm{~Hz}, 1 \mathrm{H})$ & 106.4 \\
\hline
\end{tabular}

a) Overlapped signals are reported without designating multiplicity. 


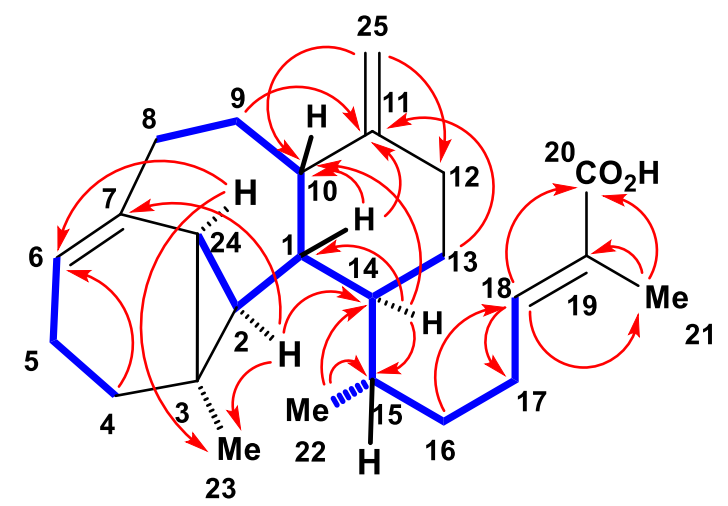

$\longrightarrow{ }^{1} \mathrm{H}-{ }^{1} \mathrm{H}$ COSY $\mathrm{HMBC}$

Figure S1. Key ${ }^{1} \mathrm{H}-{ }^{1} \mathrm{H}$ COSY and HMBC correlations of cerorubenic acid-III (1)

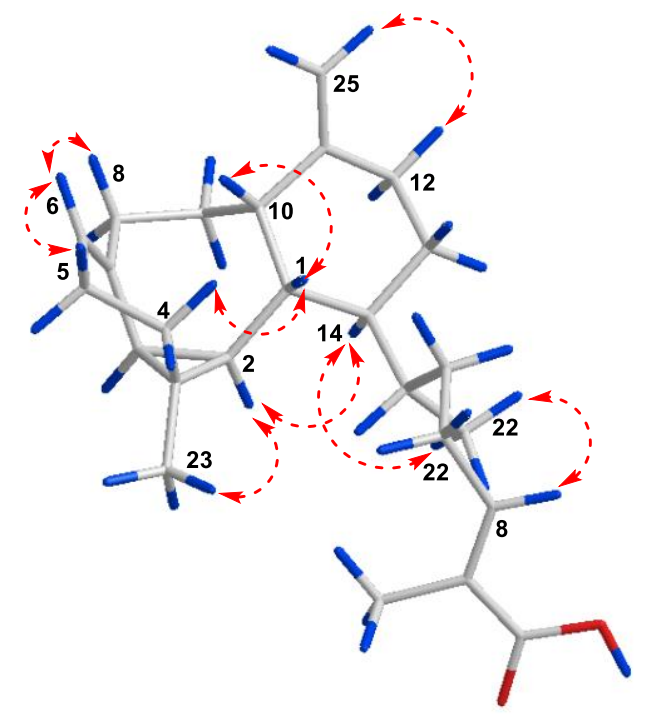

Figure S2. Key NOESY correlations of cerorubenic acid-III (1) 
Table S2. ${ }^{1} \mathrm{H}(500 \mathrm{MHz})$ and ${ }^{13} \mathrm{C}(126 \mathrm{MHz}) \mathrm{NMR}$ data of cerorubenic acid-III methyl ester (2) in $\mathrm{C}_{6} \mathrm{D}_{6}(\delta \text { in ppm, } J \text { in } \mathrm{Hz})^{a}$

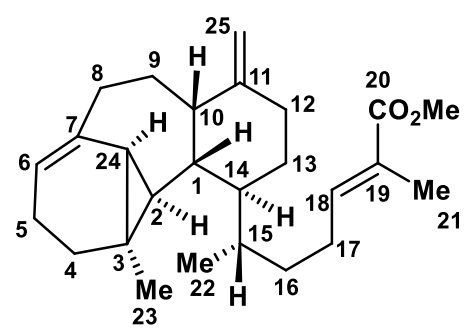

cerorubenic acid-III methyl ester (2)

\begin{tabular}{|c|c|c|}
\hline position & $\delta_{\mathrm{H}}$ & $\delta_{\mathrm{C}}$ \\
\hline 1 & $1.83(\mathrm{~m}, 1 \mathrm{H})$ & 36.3 \\
\hline 2 & $0.71(\mathrm{dd}, J=10.5,9.1 \mathrm{~Hz}, 1 \mathrm{H})$ & 31.1 \\
\hline 3 & - & 20.7 \\
\hline 4 & $1.62(\mathrm{~m}, 1 \mathrm{H}), 1.43(\mathrm{~m}, 1 \mathrm{H})$ & 28.9 \\
\hline 5 & $2.09(\mathrm{~m}, 1 \mathrm{H}), 1.77(\mathrm{~m}, 1 \mathrm{H})$ & 24.2 \\
\hline 6 & $5.71-5.65(\mathrm{~m}, 1 \mathrm{H})$ & 124.4 \\
\hline 7 & - & 140.5 \\
\hline 8 & $2.53-2.46(\mathrm{~m}, 1 \mathrm{H}), 2.15(\mathrm{~m}, 1 \mathrm{H})$ & 32.9 \\
\hline 9 & $2.22(\mathrm{~m}, 1 \mathrm{H}), 1.51(\mathrm{~m}, 1 \mathrm{H})$ & 28.6 \\
\hline 10 & $2.23(\mathrm{~m}, 1 \mathrm{H})$ & 40.6 \\
\hline 11 & - & 151.3 \\
\hline 12 & $2.19(\mathrm{~m}, 1 \mathrm{H}), 2.16(\mathrm{~m}, 1 \mathrm{H})$ & 32.8 \\
\hline 13 & $1.58(\mathrm{~m}, 1 \mathrm{H}), 1.40(\mathrm{~m}, 1 \mathrm{H})$ & 23.9 \\
\hline 14 & $1.19(\mathrm{~m}, 1 \mathrm{H})$ & 44.9 \\
\hline 15 & $1.55(\mathrm{~m}, 1 \mathrm{H})$ & 32.2 \\
\hline 16 & $1.37(\mathrm{~m}, 1 \mathrm{H}), 1.34(\mathrm{~m}, 1 \mathrm{H})$ & 32.6 \\
\hline 17 & $1.98(\mathrm{~m}, 1 \mathrm{H}), 1.91(\mathrm{~m}, 1 \mathrm{H})$ & 26.5 \\
\hline 18 & $6.93(\mathrm{dt}, J=1.5,8.0 \mathrm{~Hz}, 1 \mathrm{H})$ & 142.6 \\
\hline 19 & - & $127.7^{b}$ \\
\hline 20 & - & 168.1 \\
\hline 21 & $1.87(\mathrm{~s}, 3 \mathrm{H})$ & 12.6 \\
\hline 22 & $0.81(\mathrm{~d}, J=6.7 \mathrm{~Hz}, 3 \mathrm{H})$ & 18.1 \\
\hline 23 & $1.05(\mathrm{~s}, 3 \mathrm{H})$ & 27.3 \\
\hline 24 & $0.92(\mathrm{~d}, J=8.5 \mathrm{~Hz}, 1 \mathrm{H})$ & 24.8 \\
\hline 25 & $4.95(\mathrm{~s}, 1 \mathrm{H}), 4.89(\mathrm{~d}, J=1.8 \mathrm{~Hz}, 1 \mathrm{H})$ & 106.9 \\
\hline $\mathrm{OMe}$ & $3.45(\mathrm{~s}, 3 \mathrm{H})$ & 51.3 \\
\hline
\end{tabular}

${ }^{a)}$ Overlapped signals are reported without designating multiplicity. ${ }^{b}$ the signal of the enoate carbon 19 is obscured by solvent peaks, and it was elucidated by the correlations of H21 and C19 in the HMBC spectrum. 


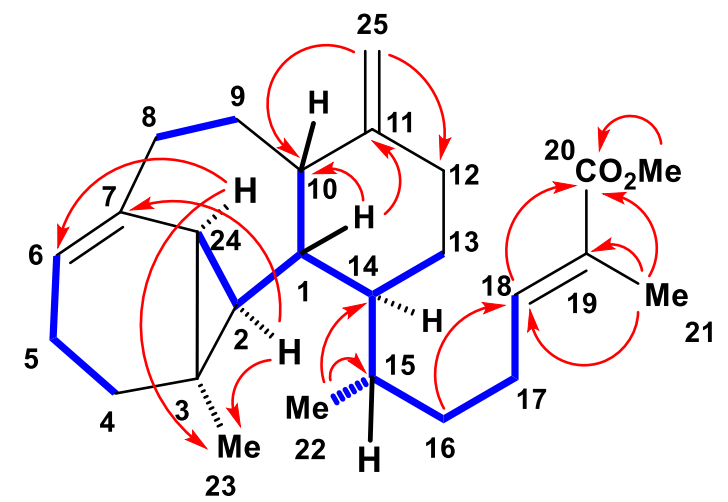

$\longrightarrow{ }^{1} \mathrm{H}-{ }^{1} \mathrm{H}$ COSY

Figure S3. Key ${ }^{1} \mathrm{H}-{ }^{1} \mathrm{H}$ COSY and HMBC correlations of cerorubenic acid-III methyl ester (2)

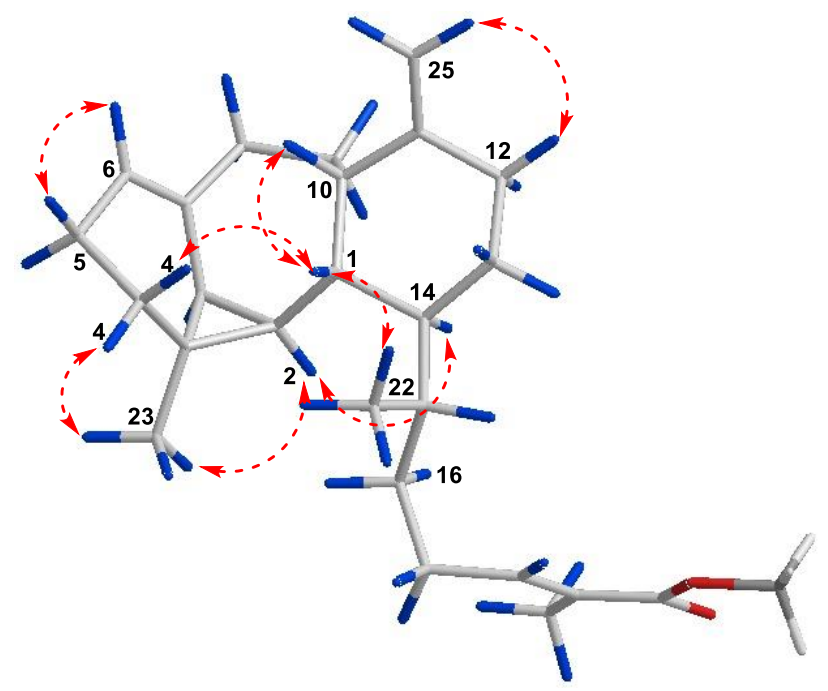

Figure S4. Key NOESY correlations of cerorubenic acid-III methyl ester (2) 
Table S3. Compared NMR data of synthetic cerorubenic acid-III methyl ester (2) between this work $\left(500 \mathrm{MHz}, \mathrm{C}_{6} \mathrm{D}_{6}\right)$ and Paquette's work $\left(300 \mathrm{MHz}, \mathrm{C}_{6} \mathrm{D}_{6}\right)$.

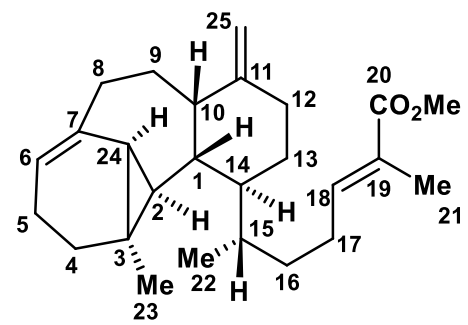

cerorubenic acid-III methyl ester (2)

\begin{tabular}{|c|c|c|c|c|c|}
\hline \multicolumn{3}{|c|}{${ }^{1} H \& p p m(J)$} & \multicolumn{3}{|c|}{${ }^{13} \mathrm{C} \& \mathrm{ppm}$} \\
\hline $\begin{array}{l}\text { this work } \\
(500 \mathrm{M})\end{array}$ & $\begin{array}{l}\text { Paquette } \\
(300 \mathrm{M})\end{array}$ & $\begin{array}{l}\text { error } \\
\text { - Paquette) }\end{array}$ & $\begin{array}{l}\text { this work } \\
(126 \mathrm{M})\end{array}$ & $\begin{array}{l}\text { Paquette } \\
\text { (75M) }\end{array}$ & $\begin{array}{c}\text { error } \\
\text { (this - Paquette) }\end{array}$ \\
\hline $6.93(\mathrm{dt}, J=1.5,8.0 \mathrm{~Hz}, 1 \mathrm{H})$ & $6.92(\mathrm{dt}, J=1.4,7.5 \mathrm{~Hz}, 1 \mathrm{H})$ & 0.01 & 168.1 & 168.1 & 0 \\
\hline $5.71-5.65(\mathrm{~m}, 1 \mathrm{H})$ & $5.70-5.67(m, 1 H)$ & 0.01 & 151.3 & 151.3 & 0 \\
\hline $4.95(\mathrm{~s}, 1 \mathrm{H})$ & $4.95(\mathrm{~s}, 1 \mathrm{H})$ & 0 & 142.6 & 142.6 & 0 \\
\hline $4.89(\mathrm{~d}, J=1.8 \mathrm{~Hz}, 1 \mathrm{H})$ & $4.88(\mathrm{~d}, J=0.8 \mathrm{~Hz}, 1 \mathrm{H})$ & 0.01 & 140.5 & 140.5 & 0 \\
\hline $3.45(\mathrm{~s}, 3 \mathrm{H})$ & $3,45(\mathrm{~s} 3 \mathrm{H})$ & 0 & $\begin{array}{l}124.4 \\
106.9\end{array}$ & $\begin{array}{l}124.4 \\
106.9\end{array}$ & $\begin{array}{l}0 \\
0\end{array}$ \\
\hline & $3.45(\mathrm{~s}, 3 \mathrm{H})$ & 0 & 51.3 & $\begin{array}{r}100.9 \\
51.2\end{array}$ & 0.1 \\
\hline $2.53-2.46(\mathrm{~m}, 1 \mathrm{H})$ & $2.55-2.48(\mathrm{~m}, 1 \mathrm{H})$ & -0.02 & $\begin{array}{l}44.9 \\
40.6\end{array}$ & $\begin{array}{l}44.9 \\
40.6\end{array}$ & $\begin{array}{l}0 \\
0\end{array}$ \\
\hline $\begin{array}{l}2.27-1.96(\mathrm{~m}, 7 \mathrm{H}) \\
1.90(\mathrm{~m}, 1 \mathrm{H}) \\
1.87(\mathrm{~s}, 3 \mathrm{H}) \\
1.83(\mathrm{dt}, \mathrm{J}=10.3,5.2 \mathrm{~Hz}, 1 \mathrm{H}) \\
1.80-1.73(\mathrm{~m}, 1 \mathrm{H})\end{array}$ & $2.34-1.72$ (series of $\mathrm{m}, 13 \mathrm{H}$ ) & & $\begin{array}{c}36.3 \\
32.9(32.859) \\
32.8(32.765) \\
32.6 \\
32.2 \\
31.1\end{array}$ & $\begin{array}{l}36.3 \\
\\
32.8 \\
32.6 \\
32.2 \\
31.1\end{array}$ & $\begin{array}{l}0 \\
0 \\
0 \\
0 \\
0\end{array}$ \\
\hline $\begin{array}{l}1.66-1.31(\mathrm{~m}, 8 \mathrm{H}) \\
1.19(\mathrm{dt}, J=6.7,4.4 \mathrm{~Hz}, 1 \mathrm{H})\end{array}$ & $\begin{array}{l}1.65-1.30(\mathrm{~m}, 8 \mathrm{H}) \\
1.23-1.17(\mathrm{~m}, 1 \mathrm{H})\end{array}$ & 0.01 & $\begin{array}{l}28.9 \\
28.6 \\
27.3 \\
\end{array}$ & $\begin{array}{l}30.0 \\
28.9 \\
28.7 \\
27.3 \\
\end{array}$ & $\begin{array}{r}0 \\
-0.1 \\
0 \\
\end{array}$ \\
\hline $1.05(\mathrm{~s}, 3 \mathrm{H})$ & $1.05(\mathrm{~s}, 3 \mathrm{H})$ & 0 & $\begin{array}{l}26.5 \\
24.8\end{array}$ & $\begin{array}{l}26.5 \\
24.8\end{array}$ & $\begin{array}{l}0 \\
0\end{array}$ \\
\hline $0.92(\mathrm{~d}, J=8.5 \mathrm{~Hz}, 1 \mathrm{H})$ & $0.92(d, J=9.7 \mathrm{~Hz}, 1 \mathrm{H})$ & 0 & 24.0 & 24.2 & 0 \\
\hline $0.81(\mathrm{~d}, J=6.7 \mathrm{~Hz}, 3 \mathrm{H})$ & $0.81(\mathrm{~d}, J=6.7 \mathrm{~Hz}, 3 \mathrm{H})$ & 0 & 23.9 & 23.9 & 0 \\
\hline $0.71(\mathrm{dd}, J=10.5,9.1 \mathrm{~Hz}, 1 \mathrm{H})$ & $0.70(\mathrm{dd}, J=10.2,9.4 \mathrm{~Hz}, 1 \mathrm{H})$ & 0.01 & $\begin{array}{l}20.7 \\
18.1 \\
12.6\end{array}$ & $\begin{array}{l}20.7 \\
18.0 \\
12.5\end{array}$ & $\begin{array}{r}0 \\
0.1 \\
0.1\end{array}$ \\
\hline
\end{tabular}

\section{Note:}

The optical rotation of the synthetic 2 in this work $\left(\left([\alpha]_{\mathrm{D}}^{25}=-50\left(c=1.0, \mathrm{CH}_{2} \mathrm{Cl}_{2}\right)\right.\right.$, $\left.[\alpha]_{\mathrm{D}}^{25}=-28\left(c=0.054, \mathrm{CHCl}_{3}\right)\right)$ was closed to that of Naya's work $\left([\alpha]_{\mathrm{D}}^{24}=-60(c=\right.$ $\left.\left.0.001, \mathrm{CHCl}_{3}\right)\right),{ }^{1}$ and Paquette's work $\left([\alpha]_{\mathrm{D}}^{20}=-124\left(c=0.001, \mathrm{CHCl}_{3}\right)\right) .{ }^{2}$ The ${ }^{1} \mathrm{H}$ spectra of 2 in this work were identical to those of Paquette's work. The ${ }^{13} \mathrm{C}$ NMR spectra of $\mathbf{2}$ in this work were almost identical to those of Paquette's work except for the chemical shift of only one carbon (32.9 in this work and 30.0 in Paquette's work) as shown in the Table S3.

Our efforts to get the copies of the original spectra of compound $\mathbf{2}$ from Prof. Paquette or Prof. Naya have all failed to date, because they have retired for a long time. 
In turn, we confirmed our structure of natural product (1) and its methyl ester (2) to be the reported one based on the comprehensive analysis of ${ }^{1} \mathrm{H},{ }^{13} \mathrm{C}$, dept-135, HSQC, ${ }^{1} \mathrm{H}-$ ${ }^{1} \mathrm{H}$ COSY, HMBC and NOESY spectra (Table S1-S2 and Figure S1-S4). We confirmed that chemical shift 32.9 is for C8 and chemical shift 32.8 is for $\mathrm{C} 12$ in compound 2. Further analysis of the carbon spectrum in table S3, we preferred to believe that the carbon 32.8 (in Paquette's work) was an overlapped single of two carbons (32.859 and 32.765 in this work), because of their imperceptible distance (less than $0.1 \mathrm{ppm}$ ) and the probably different dispersion of the $300 \mathrm{MHz}-\mathrm{NMR}$ machine in more than 20 years ago. Meanwhile, the carbon 30.0 (in Paquette's work) was likely a mistaken assignment of grease ${ }^{3}$ (a frequently observed impurity in the spectrum, Figure S5) by the author because of the scarcity or inadequate purity of the sample.

\begin{tabular}{|c|c|c|c|c|c|c|c|c|c|c|c|c|c|}
\hline & carbon & THF- $d_{8}$ & $\mathrm{CD}_{2} \mathrm{Cl}_{2}$ & $\mathrm{CDCl}_{3}$ & toluene- $d_{8}$ & $\mathrm{C}_{6} \mathrm{D}_{6}$ & $\mathrm{C}_{6} \mathrm{D}_{5} \mathrm{Cl}$ & $\left(\mathrm{CD}_{3}\right)_{2} \mathrm{CO}$ & $\left(\mathrm{CD}_{3}\right)_{2} \mathrm{SO}$ & $\mathrm{CD}_{3} \mathrm{CN}$ & TFE- $d_{3}$ & $\mathrm{CD}_{3} \mathrm{OD}$ & $\mathrm{D}_{2} \mathrm{O}$ \\
\hline solvent signals & & $\begin{array}{l}67.21 \\
25.31\end{array}$ & 53.84 & 77.16 & $\begin{array}{c}137.48 \\
128.87 \\
127.96 \\
125.13 \\
20.43\end{array}$ & 128.06 & $\begin{array}{l}134.19 \\
129.26 \\
128.25 \\
125.96\end{array}$ & $\begin{array}{r}29.84 \\
206.26\end{array}$ & 39.52 & $\begin{array}{c}1.32 \\
118.26\end{array}$ & $\begin{array}{c}61.50 \\
126.28\end{array}$ & 49.00 & \\
\hline \multirow{2}{*}{ acetic acid } & $\mathrm{CO}$ & 171.69 & 175.85 & 175.99 & 175.30 & 175.82 & 175.67 & 172.31 & 171.93 & 173.21 & 177.96 & 175.11 & 177.21 \\
\hline & $\mathrm{CH}_{3}$ & 20.13 & 20.91 & 20.81 & 20.27 & 20.37 & 20.40 & 20.51 & 20.95 & 20.73 & 20.91 & 20.56 & 21.03 \\
\hline \multirow[t]{2}{*}{ acetone } & $\mathrm{CO}$ & 204.19 & 206.78 & 207.07 & 204.00 & 204.43 & 204.83 & 205.87 & 206.31 & 207.43 & 32.35 & 209.67 & 215.94 \\
\hline & $\mathrm{CH}_{3}$ & 30.17 & 31.00 & 30.92 & 30.03 & 30.14 & 30.12 & 30.60 & 30.56 & 30.91 & 214.98 & 30.67 & 30.89 \\
\hline \multirow{2}{*}{ acetonitrile } & $\mathrm{CN}$ & 116.79 & 116.92 & 116.43 & 115.76 & 116.02 & 115.93 & 117.60 & 117.91 & 118.26 & 118.95 & 118.06 & 119.68 \\
\hline & $\mathrm{CH}_{3}$ & 0.45 & 2.03 & 1.89 & 0.03 & 0.20 & 0.63 & 1.12 & 1.03 & 1.79 & 1.00 & 0.85 & 1.47 \\
\hline \multirow{3}{*}{ tert -butyl alcohol } & $\mathrm{CH}$ & 128.84 & 128.68 & 128.37 & 128.57 & 128.62 & 128.38 & 129.15 & 128.30 & 129.32 & 129.84 & 129.34 & \\
\hline & $\left(\mathrm{CH}_{3}\right)_{3} \mathrm{C}$ & 67.50 & 69.11 & 69.15 & 68.12 & 68.19 & 68.19 & 68.13 & 66.88 & 68.74 & 72.35 & 69.40 & 70.36 \\
\hline & $\left(\mathrm{CH}_{3}\right)_{3} \mathrm{C}$ & 30.57 & 31.46 & 31.25 & 30.49 & 30.47 & 31.13 & 30.72 & 30.38 & 30.68 & 31.07 & 30.91 & 30.29 \\
\hline carbon dioxide & $\mathrm{CO}_{2}$ & 125.69 & 125.26 & 124.99 & 124.86 & 124.76 & 126.08 & 125.81 & 124.21 & 125.89 & 126.92 & 126.31 & \\
\hline carbon disulfide & $\mathrm{CS}_{2}$ & 193.37 & 192.95 & 192.83 & 192.71 & 192.69 & 192.49 & 193.58 & 192.63 & 193.60 & 196.26 & 193.82 & 197.25 \\
\hline carbon tetrachloride & $\mathrm{CCl}_{4}^{2}$ & 96.89 & 96.52 & 96.34 & 96.57 & 96.44 & 96.38 & 96.65 & 95.44 & 96.68 & 97.74 & 97.21 & 96.73 \\
\hline chloroform & $\mathrm{CH}$ & 79.24 & 77.99 & 77.36 & 77.89 & 77.79 & 77.67 & 79.19 & 79.16 & 79.17 & 78.83 & 79.44 & \\
\hline 18-crown-6 & $\mathrm{CH}_{2}$ & 71.34 & 70.47 & 70.55 & 70.86 & 70.59 & 70.55 & 71.25 & 69.85 & 71.22 & 70.80 & 71.47 & 70.14 \\
\hline cyclohexane & $\mathrm{CH}_{2}$ & 27.58 & 27.38 & 26.94 & 27.31 & 27.23 & 26.99 & 27.51 & 26.33 & 27.63 & 28.34 & 27.96 & \\
\hline 1,2-dichloroethane & $\mathrm{CH}_{2}$ & 44.64 & 44.35 & 43.50 & 43.40 & 43.59 & 43.60 & 45.25 & 45.02 & 45.54 & 45.28 & 45.11 & \\
\hline dichloromethane & $\mathrm{CH}_{2}$ & 54.67 & 54.24 & 53.52 & 53.47 & 53.46 & 53.54 & 54.95 & 54.84 & 55.32 & 54.46 & 54.78 & \\
\hline \multirow[t]{2}{*}{ diethyl ether } & $\mathrm{CH}_{3}$ & 15.49 & 15.44 & 15.20 & 15.47 & 15.46 & 15.35 & 15.78 & 15.12 & 15.63 & 15.33 & 15.46 & 14.77 \\
\hline & $\mathrm{CH}_{2}$ & 66.14 & 66.11 & 65.91 & 65.94 & 65.94 & 65.79 & 66.12 & 62.05 & 66.32 & 67.55 & 66.88 & 66.42 \\
\hline \multirow[t]{3}{*}{ diglyme } & $\mathrm{CH}_{3}$ & 58.72 & 58.95 & 59.01 & 58.62 & 58.66 & 58.42 & 58.77 & 57.98 & 58.90 & 59.40 & 59.06 & 58.67 \\
\hline & $\mathrm{CH}_{2}$ & 71.17 & 70.70 & 70.51 & 70.92 & 70.87 & 70.56 & 71.03 & 69.54 & 70.99 & 73.05 & 71.33 & 70.05 \\
\hline & $\mathrm{CH}_{2}$ & 72.72 & 72.25 & 71.90 & 72.39 & 72.35 & 72.07 & 72.63 & 71.25 & 72.63 & 71.33 & 72.92 & 71.63 \\
\hline \multirow[t]{3}{*}{ dimethylformamide } & $\mathrm{CH}$ & 161.96 & 162.57 & 162.62 & 161.93 & 162.13 & 162.01 & 162.79 & 162.29 & 163.31 & 166.01 & 164.73 & 165.53 \\
\hline & $\mathrm{CH}_{3}$ & 35.65 & 36.56 & 36.50 & 35.22 & 35.25 & 35.45 & 36.15 & 35.73 & 36.57 & 37.76 & 36.89 & 37.54 \\
\hline & $\mathrm{CH}_{3}$ & 30.70 & 31.39 & 31.45 & 30.64 & 30.72 & 30.71 & 31.03 & 30.73 & 31.32 & 30.96 & 31.61 & 32.03 \\
\hline 1,4-dioxane & $\mathrm{CH}_{2}$ & 67.65 & 67.47 & 67.14 & 67.17 & 67.16 & 66.95 & 67.60 & 66.36 & 67.72 & 68.52 & 68.11 & 67.19 \\
\hline \multirow[t]{2}{*}{ DME } & $\mathrm{CH}_{3}$ & 58.72 & 59.02 & 59.08 & 58.63 & 58.68 & 58.31 & 58.45 & 58.03 & 58.89 & 59.52 & 59.06 & 58.67 \\
\hline & $\mathrm{CH}_{2}$ & 72.58 & 72.24 & 71.84 & 72.25 & 72.21 & 71.81 & 72.47 & 71.17 & 72.47 & 72.87 & 72.72 & 71.49 \\
\hline \multirow{3}{*}{$\begin{array}{l}\text { ethane } \\
\text { ethanol }\end{array}$} & $\mathrm{CH}_{3}$ & 6.79 & 6.91 & 6.89 & 6.94 & 6.96 & 6.91 & 6.88 & 6.61 & 6.99 & 7.01 & 6.98 & \\
\hline & $\mathrm{CH}_{3}$ & 18.90 & 18.69 & 18.41 & 18.78 & 18.72 & 18.55 & 18.89 & 18.51 & 18.80 & 18.11 & 18.40 & 17.47 \\
\hline & $\mathrm{CH}_{2}$ & 57.60 & 58.57 & 58.28 & 57.81 & 57.86 & 57.63 & 57.72 & 56.07 & 57.96 & 59.68 & 58.26 & 58.05 \\
\hline \multirow[t]{4}{*}{ ethyl acetate } & $\mathrm{CH}_{3} \mathrm{CO}$ & 20.45 & 21.15 & 21.04 & 20.46 & 20.56 & 20.50 & 20.83 & 20.68 & 21.16 & 21.18 & 20.88 & 21.15 \\
\hline & $\mathrm{CO}$ & 170.32 & 171.24 & 171.36 & 170.02 & 170.44 & 170.20 & 170.96 & 170.31 & 171.68 & 175.55 & 172.89 & 175.26 \\
\hline & $\mathrm{CH}_{2}$ & 60.30 & 60.63 & 60.49 & 60.08 & 60.21 & 60.06 & 60.56 & 59.74 & 60.98 & 62.70 & 61.50 & 62.32 \\
\hline & $\mathrm{CH}_{3}$ & 14.37 & 14.37 & 14.19 & 14.23 & 14.19 & 14.07 & 14.50 & 14.40 & 14.54 & 14.36 & 14.49 & 13.92 \\
\hline ethylene & $\mathrm{CH}_{2}$ & 123.09 & 123.20 & 123.13 & 122.92 & 122.96 & 122.95 & 123.47 & 123.52 & 123.69 & 124.08 & 123.46 & \\
\hline ethylen & $\mathrm{CH}_{2}$ & 64.35 & 6408 & 6370 & 6420 & 6434 & 6403 & 6426 & 62.76 & 64.22 & 64.87 & 64.30 & 63.17 \\
\hline $\mathrm{H}$ grease $^{5}$ & $\mathrm{CH}_{2}$ & 30.45 & 30.14 & 29.71 & 30.31 & 30.22 & 30.11 & & & & & & \\
\hline
\end{tabular}

Figure S5. NMR chemical shifts of trace impurities 


\section{References:}

[1]. Tempesta, M. S.; Iwashita, T.; Miyamoto, F.; Yoshihara, K.; Naya, Y. A New Class of Sesterterpenoids from the Secretion of Ceroplastes Rubens (Coccidae). J. Chem. Soc., Chem. Commun. 1983, 1182-1183.

[2]. Paquette, L. A.; Dyck, B. P., Studies Directed toward the Total Synthesis of Cerorubenic Acid-III. 5. A radical Cyclization Route Leading to the Methyl Ester of the Natural Isomer. J. Am. Chem. Soc. 1998, 120, 5953-5960.

[3]. Fulmer, G. R.; Miller, A. J. M.; Sherden, N. H.; Gottlieb, H. E.; Nudelman, A.; Stoltz, B. M.; Bercaw, J. E.; Goldberg, K. I. NMR Chemical Shifts of Trace Impurities: Common Laboratory Solvents, Organics, and Gases in Deuterated Solvents Relevant to the Organometallic Chemist. Organometallics 2010, 29, 2176-2179. 


\section{X-ray Crystallographic Data}

\section{X-Ray data for 25: (CCDC 2052612)}
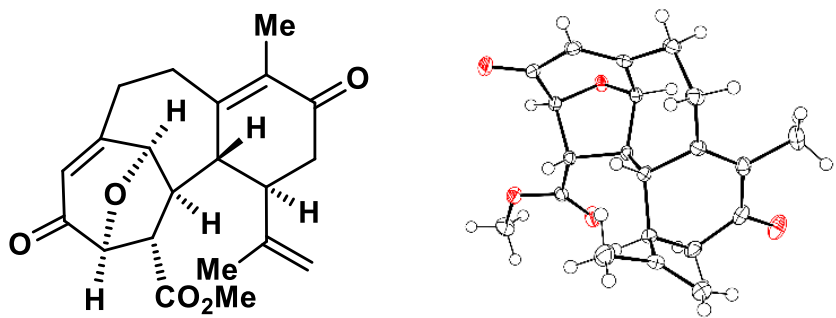

Crystal data and structure refinement for $\mathbf{2 5}$.

Identification code

A

Empirical formula

$\mathrm{C}_{21} \mathrm{H}_{24} \mathrm{O}_{5}$

Formula weight

356.40

Temperature/K

150.0

Crystal system

triclinic

Space group

P-1

$\mathrm{a} / \AA$

$8.5244(15)$

$\mathrm{b} / \AA$

$9.7758(16)$

$\mathrm{c} / \AA$

$11.6492(16)$

$\alpha /^{\circ}$

$80.394(5)$

$\beta /{ }^{\circ}$

$70.076(4)$

$\gamma /{ }^{\circ}$

$72.081(5)$

Volume $/ \AA^{3}$

$866.3(2)$

\section{Z}

2

$\rho_{\text {calc }} / \mathrm{cm}^{3}$

1.366

$\mu / \mathrm{mm}^{-1}$

0.097

$\mathrm{F}(000)$

380.0

Crystal size $/ \mathrm{mm}^{3}$

$? \times ? \times ?$

Radiation

$2 \Theta$ range for data collection ${ }^{\circ}$

$\operatorname{MoK} \alpha(\lambda=0.71073)$

Index ranges

Reflections collected

5.28 to 61.136

$-12 \leq \mathrm{h} \leq 12,-13 \leq \mathrm{k} \leq 13,-16 \leq 1 \leq 16$

23215

Independent reflections

$5247\left[\mathrm{R}_{\text {int }}=0.0375, \mathrm{R}_{\text {sigma }}=0.0395\right]$

Data/restraints/parameters

$5247 / 0 / 239$

Goodness-of-fit on $\mathrm{F}^{2}$

1.036

Final $\mathrm{R}$ indexes $[\mathrm{I}>=2 \sigma(\mathrm{I})]$

$\mathrm{R}_{1}=0.0480, \mathrm{wR}_{2}=0.1096$

Final $\mathrm{R}$ indexes [all data]

$\mathrm{R}_{1}=0.0672, \mathrm{wR}_{2}=0.1175$

Largest diff. peak/hole / e $\AA^{-3}$

$0.40 /-0.20$

Identification code

A 
X-Ray data for 29: (CCDC 2052613)
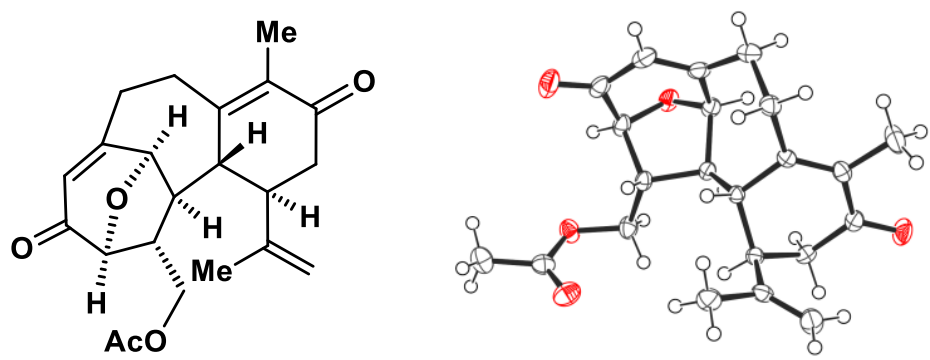

Crystal data and structure refinement for 29.

Identification code

Empirical formula

Formula weight

Temperature/K

Crystal system

Space group

$\mathrm{a} / \AA$

$\mathrm{b} / \AA$

$\mathrm{c} / \AA$

$\alpha /{ }^{\circ}$

$\beta /{ }^{\circ}$

$\gamma /{ }^{\circ}$

Volume $/ \AA^{3}$

Z

$\rho_{\text {calc } \mathrm{g} / \mathrm{cm}^{3}}$

$\mu / \mathrm{mm}^{-1}$

$\mathrm{F}(000)$

Crystal size $/ \mathrm{mm}^{3}$

Radiation

$2 \Theta$ range for data collection $/{ }^{\circ}$

Index ranges

Reflections collected

Independent reflections

Data/restraints/parameters

Goodness-of-fit on $\mathrm{F}^{2}$

Final R indexes $[\mathrm{I}>=2 \sigma(\mathrm{I})]$

Final $\mathrm{R}$ indexes [all data]

Largest diff. peak/hole / e $\AA^{-3}$

Flack parameter
A

$\mathrm{C}_{22} \mathrm{H}_{26} \mathrm{O}_{5}$

370.43

150.0

monoclinic

$\mathrm{P} 21$

$7.2248(10)$

19.750(3)

$13.3716(19)$

90

$94.210(5)$

90

$1902.8(5)$

4

1.293

0.739

792.0

$0.4 \times 0.26 \times 0.19$

$\mathrm{CuK} \alpha(\lambda=1.54178)$

6.628 to 136.46

$-8 \leq \mathrm{h} \leq 8,-23 \leq \mathrm{k} \leq 23,-16 \leq 1 \leq 16$

17142

$6848\left[\mathrm{R}_{\text {int }}=0.0243, \mathrm{R}_{\text {sigma }}=0.0286\right]$

$6848 / 1 / 493$

1.038

$\mathrm{R}_{1}=0.0330, \mathrm{wR}_{2}=0.0833$

$\mathrm{R}_{1}=0.0340, \mathrm{wR}_{2}=0.0843$

$0.16 /-0.17$

$0.11(9)$ 
X-Ray data for 60: (CCDC 1869291) (ellipsoids at the 50\% probability level)
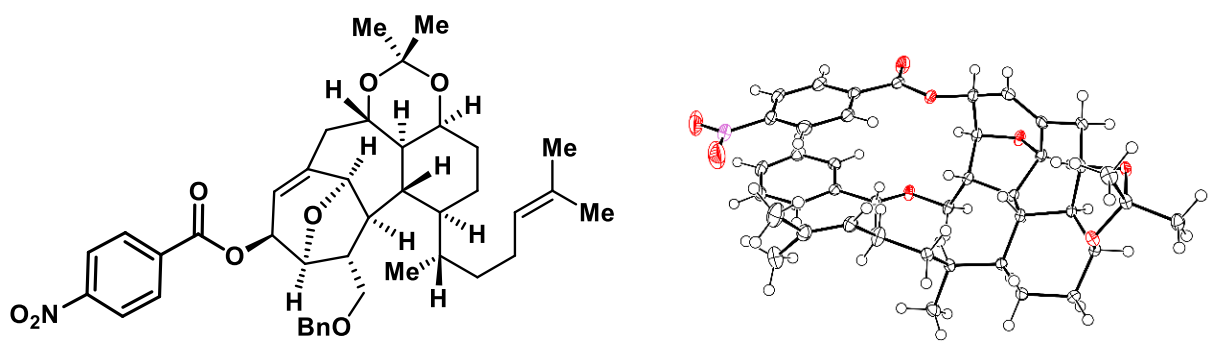

Crystals suitable for X-ray analysis were obtained from dis-solving $\mathbf{6 0}$ in $\mathrm{CH}_{2} \mathrm{Cl}_{2}$ followed by the addition of hexane, and then allowing the solvent to evaporate slowly.

Identification code

Empirical formula

Formula weight

Temperature/K

Crystal system

Space group

$\mathrm{a} / \AA$

$\mathrm{b} / \AA$

$\mathrm{c} / \AA$

$\alpha /^{\circ}$

$\beta /{ }^{\circ}$

$\gamma /{ }^{\circ}$

Volume $/ \AA^{3}$

Z

$\rho_{\text {calc }} / \mathrm{cm}^{3}$

$\mu / \mathrm{mm}^{-1}$

$\mathrm{F}(000)$

Crystal size $/ \mathrm{mm}^{3}$

Radiation

$2 \Theta$ range for data collection ${ }^{\circ}$

Index ranges

Reflections collected

Independent reflections

Data/restraints/parameters

Goodness-of-fit on $\mathrm{F}^{2}$

Final $\mathrm{R}$ indexes $[\mathrm{I}>=2 \sigma(\mathrm{I})]$

Final $\mathrm{R}$ indexes [all data]

Largest diff. peak/hole / e $\AA^{-3}$

Flack parameter
A

$\mathrm{C}_{41} \mathrm{H}_{51} \mathrm{NO}_{8}$

685.82

100

monoclinic

$\mathrm{P} 21$

9.855(3)

13.243(3)

$13.890(5)$

90

$101.25(2)$

90

1777.9(9)

2

1.281

0.088

736.0

$0.52 \times 0.5 \times 0.48$

$\operatorname{MoK} \alpha(\lambda=0.71073)$

4.668 to 55.244

$-12 \leq \mathrm{h} \leq 12,-17 \leq \mathrm{k} \leq 17,-18 \leq 1 \leq 18$

25208

$8135\left[\mathrm{R}_{\text {int }}=0.0339, \mathrm{R}_{\text {sigma }}=0.0353\right]$

$8135 / 1 / 457$

1.086

$\mathrm{R}_{1}=0.0567, \mathrm{wR}_{2}=0.1443$

$\mathrm{R}_{1}=0.0601, \mathrm{wR}_{2}=0.1471$

$0.27 /-0.22$

$-0.2(10)$ 


\section{III. ${ }^{1} \mathrm{H}$ and ${ }^{13} \mathrm{C}$ NMR Spectra}

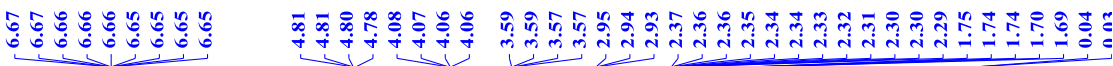<smiles>C=C(C)C1CC=C([N+](=O)[O-])C(=O)[C@@H]1COC</smiles>

15

('H-NMR, $\mathrm{CDCl}_{3}, 500 \mathrm{MHz}$ )

$\frac{9}{2}$

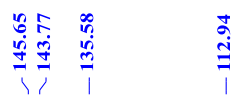

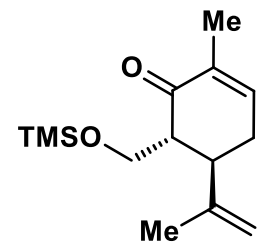

15

$\left({ }^{13} \mathrm{C}-\mathrm{NMR}, \mathrm{CDCl}_{3}, 126 \mathrm{MHz}\right)$

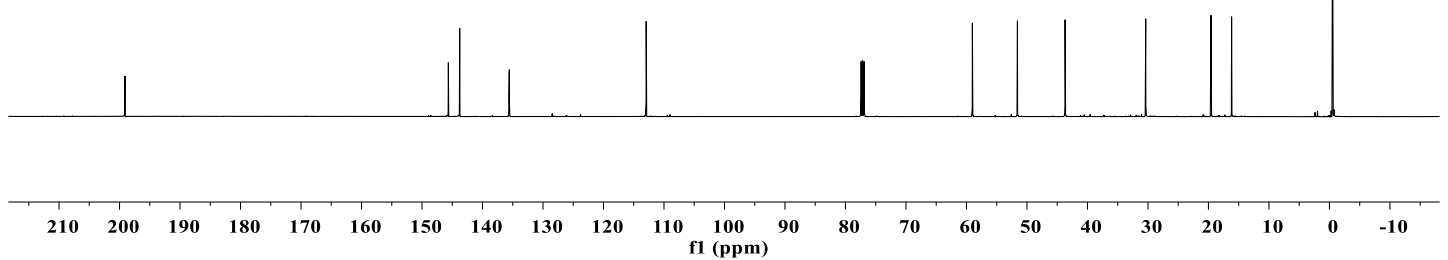



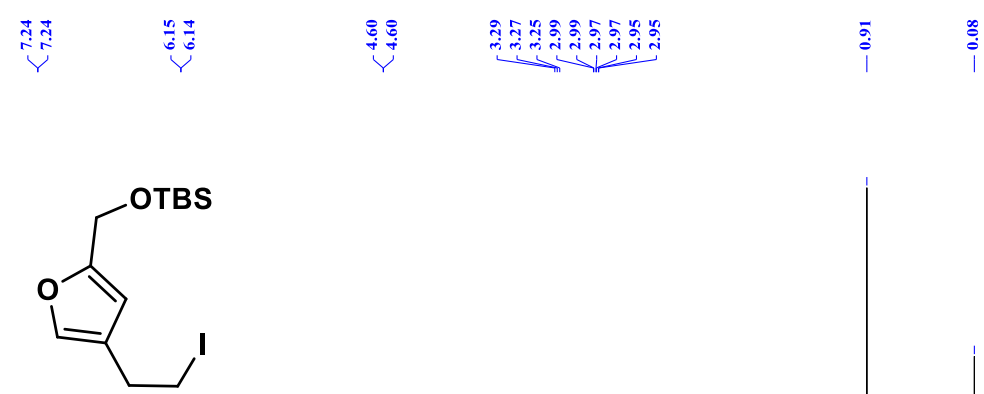

18

( ${ }^{1} \mathrm{H}-\mathrm{NMR}, \mathrm{CDCl}_{3}, 400 \mathrm{MHz}$ )
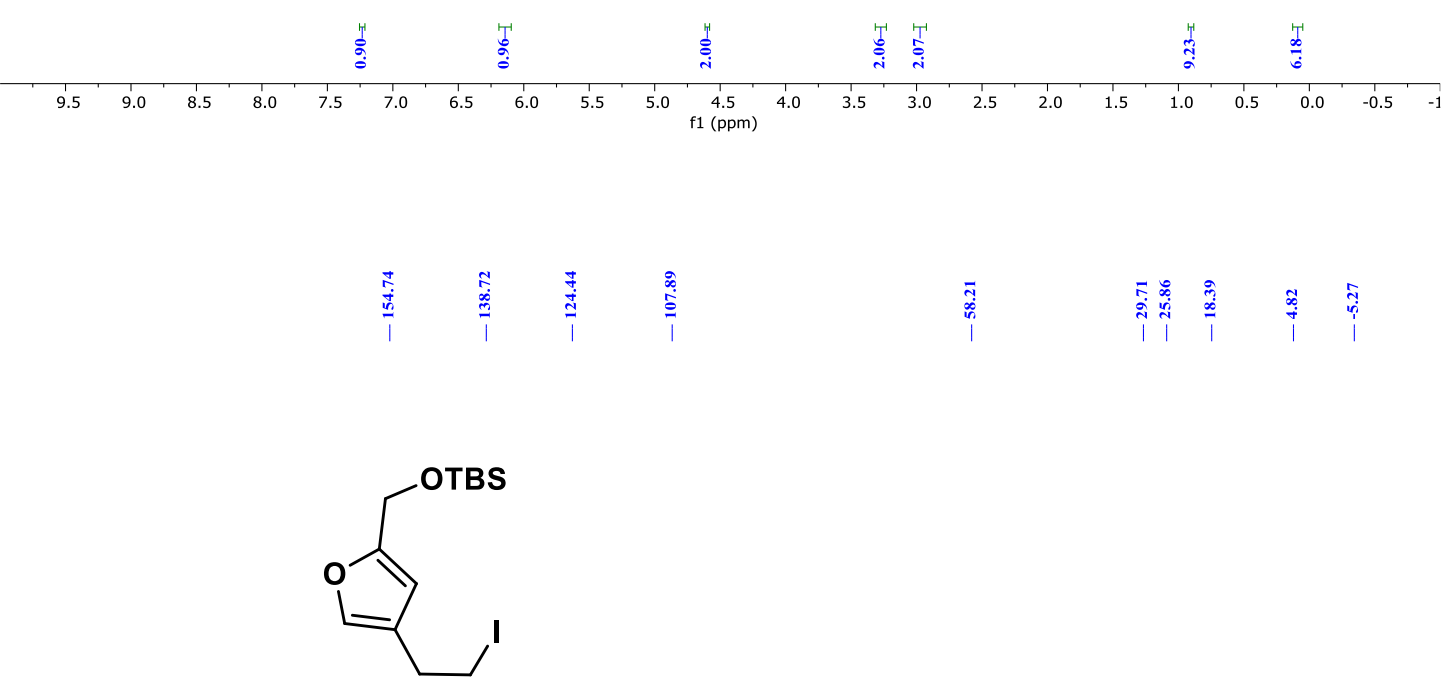

18

$\left({ }^{13} \mathrm{H}-\mathrm{NMR}, \mathrm{CDCl}_{3}, 101 \mathrm{MHz}\right)$

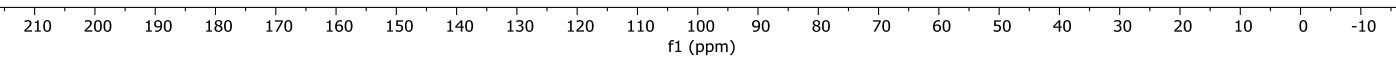




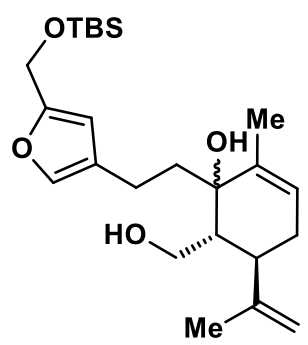

$19 a$

$\left({ }^{1} \mathrm{H}-\mathrm{NMR}, \mathrm{CDCl}_{3}, 500 \mathrm{MHz}\right)$

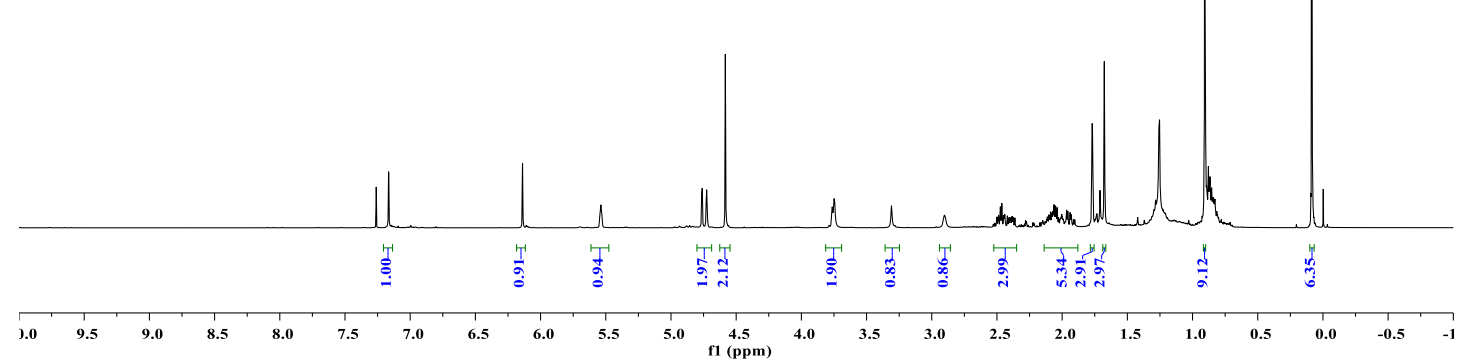

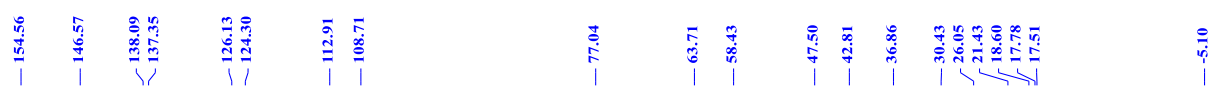

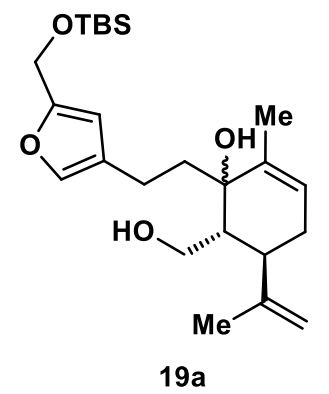

$\left({ }^{13} \mathrm{C}-\mathrm{NMR}, \mathrm{CDCl}_{3}, 126 \mathrm{MHz}\right)$

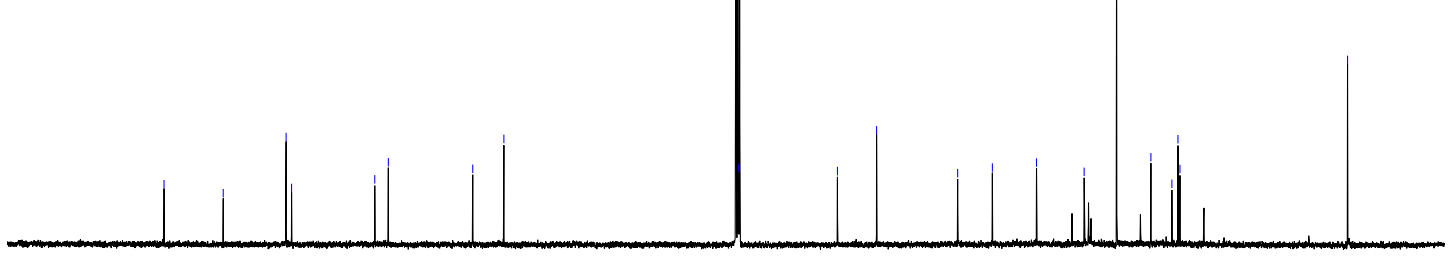

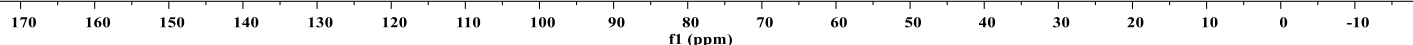




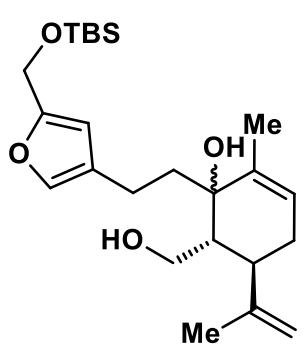

$19 b$

(' $\mathrm{H}-\mathrm{NMR}, \mathrm{CDCl}_{3}, 500 \mathrm{MHz}$ )

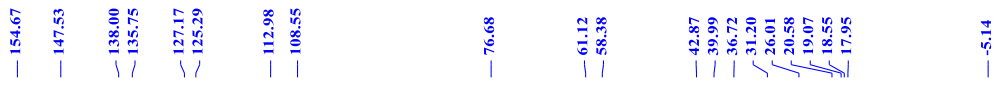<smiles>C=C(C)[C@H]1CC=C(C)[C@](O)(CCc2coc(CO[Sb])c2)[C@@H]1CO</smiles>

$19 b$

$\left({ }^{13} \mathrm{C}-\mathrm{NMR}, \mathrm{CDCl}_{3}, 126 \mathrm{MHz}\right)$

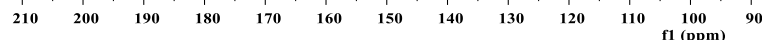




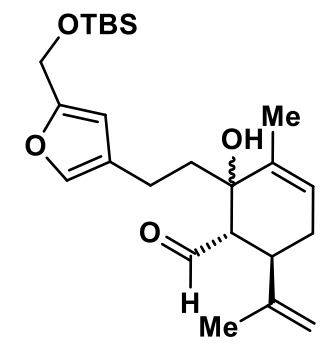

('H-NMR, $\mathrm{CDCl}_{3}, 500 \mathrm{MHz}$ )

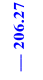<smiles>C=C(C)C1CC=C(C)C(O)(CCc2coc(COC(C)(C)C)c2)C1C(C)=O</smiles>

20a

$\left({ }^{13} \mathrm{C}-\mathrm{NMR}, \mathrm{CDCl}_{3}, 126 \mathrm{MHz}\right)$

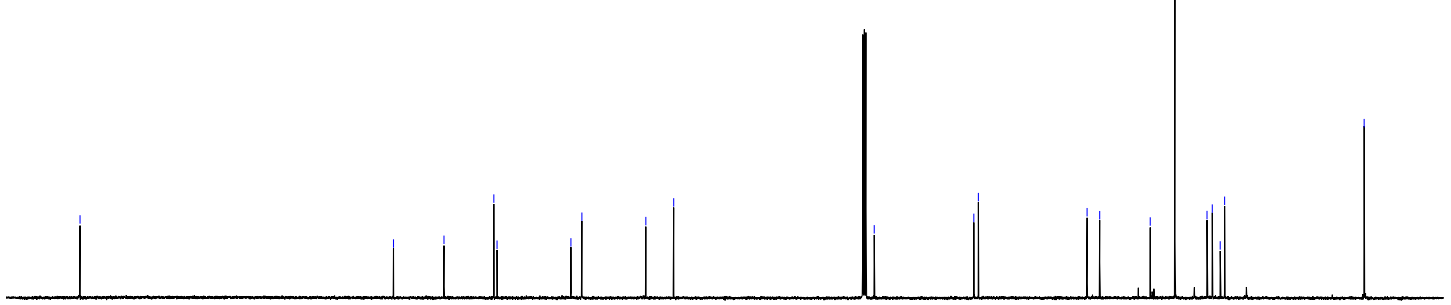

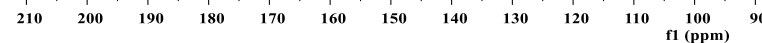




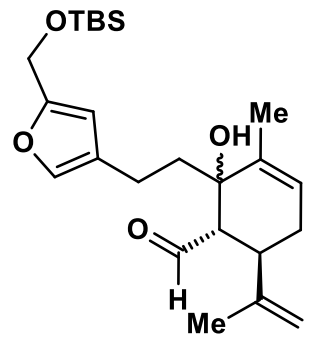

$20 \mathrm{~b}$

$\left({ }^{1} \mathrm{H}-\mathrm{NMR}, \mathrm{CDCl}_{3}, 500 \mathrm{MHz}\right)$

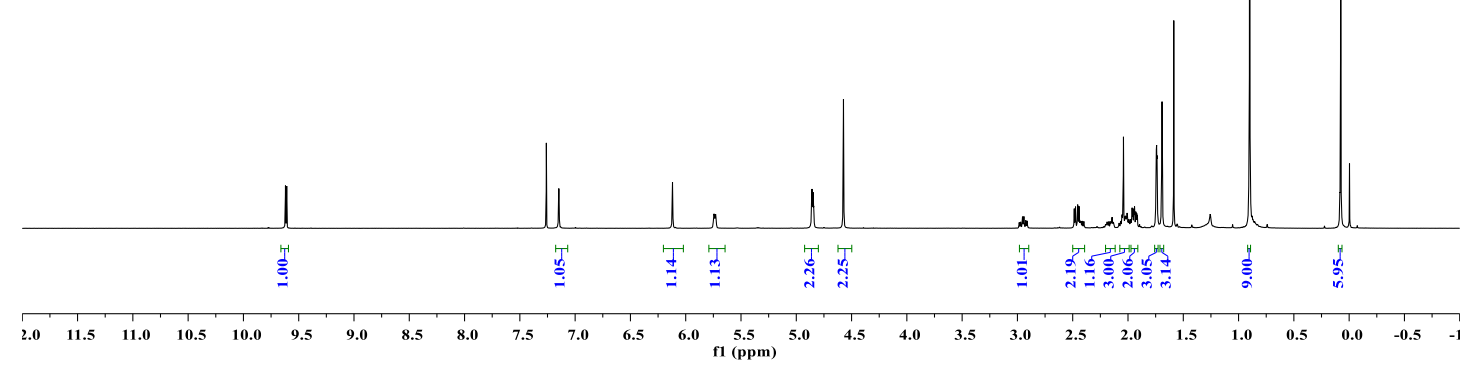

\ัָ

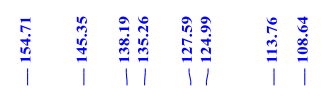<smiles>C=C(C)C1CC=C(C)C(O)(CCc2coc(COC(C)(C)C)c2)[C@H]1C(=O)O</smiles>

$20 \mathrm{~b}$

$\left({ }^{13} \mathrm{C}-\mathrm{NMR}, \mathrm{CDCl}_{3}, 126 \mathrm{MHz}\right)$

$\begin{array}{lllllllllllll}210 & 200 & 190 & 180 & 170 & 160 & 150 & 140 & 130 & 120 & 110 & \begin{array}{c}100 \\ \text { f } 1(\mathrm{ppm})\end{array} & 90\end{array}$ 


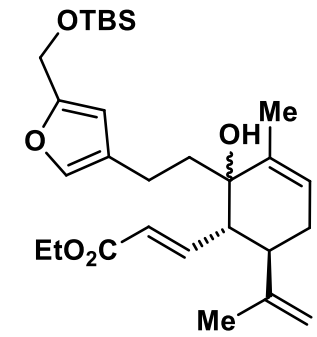

21a

( ${ }^{1} \mathrm{H}-\mathrm{NMR}, \mathrm{CDCl}_{3}, 400 \mathrm{MHz}$ )

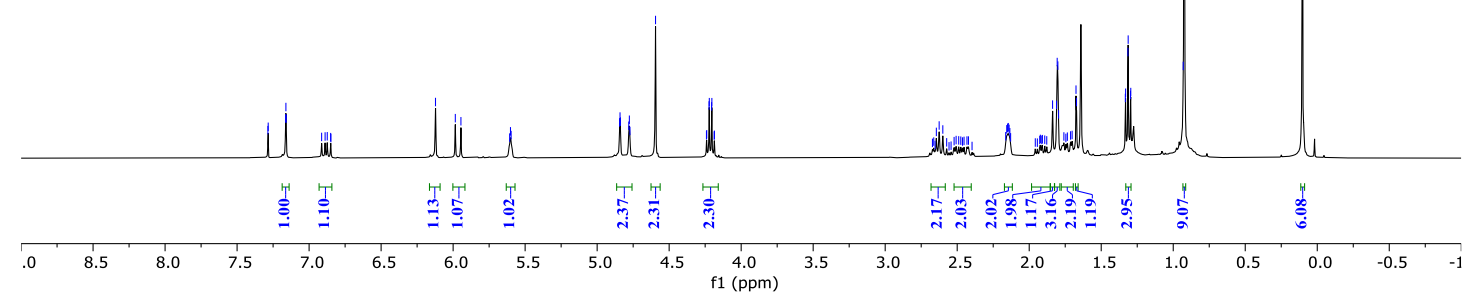

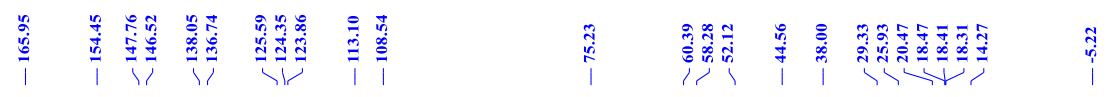

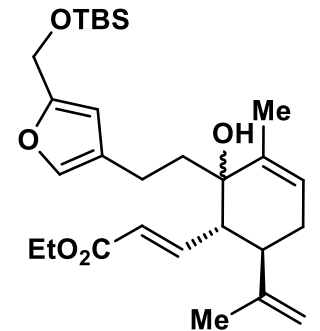

21a

$\left({ }^{13} \mathrm{C}-\mathrm{NMR}, \mathrm{CDCl}_{3}, 101 \mathrm{MHz}\right)$

$\begin{array}{lllllllllllll}210 & 200 & 190 & 180 & 170 & 160 & 150 & 140 & 130 & 120 & 110 & 100 & 90 \\ & & & & & & & & & & & & \end{array}$ 


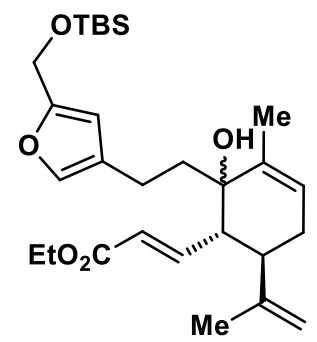

21b

( ${ }^{1} \mathrm{H}-\mathrm{NMR}, \mathrm{CDCl}_{3}, 400 \mathrm{MHz}$ )

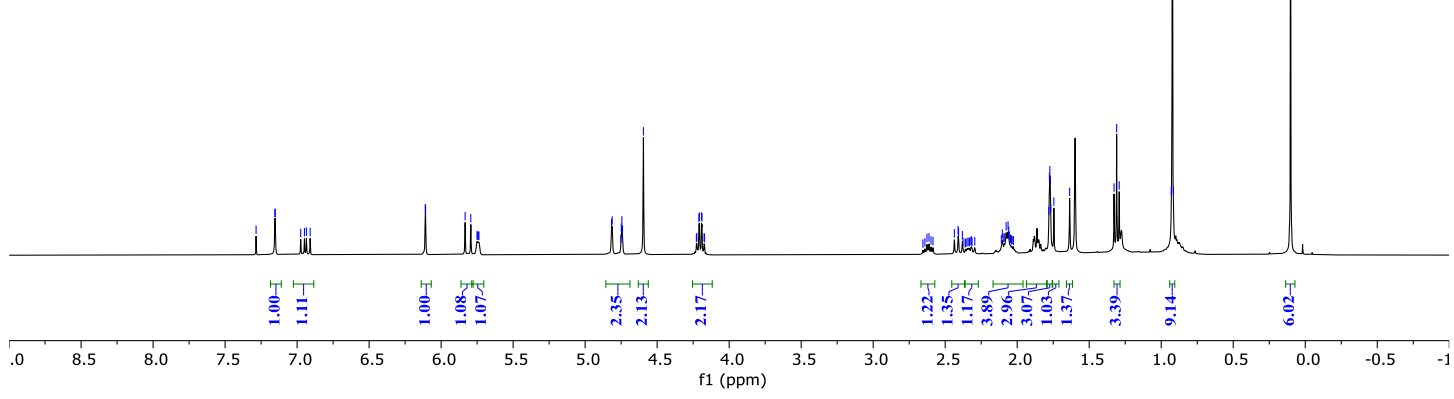

\begin{tabular}{|c|c|c|c|c|c|}
\hline 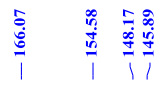 & 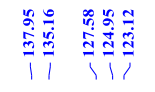 & 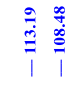 & 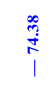 & $\prod_{0}^{0}$ & 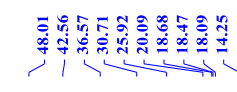 \\
\hline
\end{tabular}

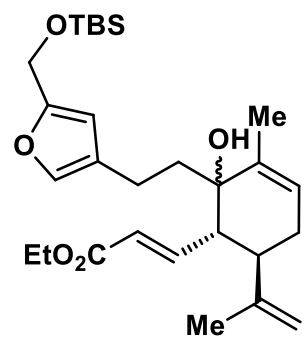

21b

$\left({ }^{13} \mathrm{C}-\mathrm{NMR}, \mathrm{CDCl}_{3}, 101 \mathrm{MHz}\right)$

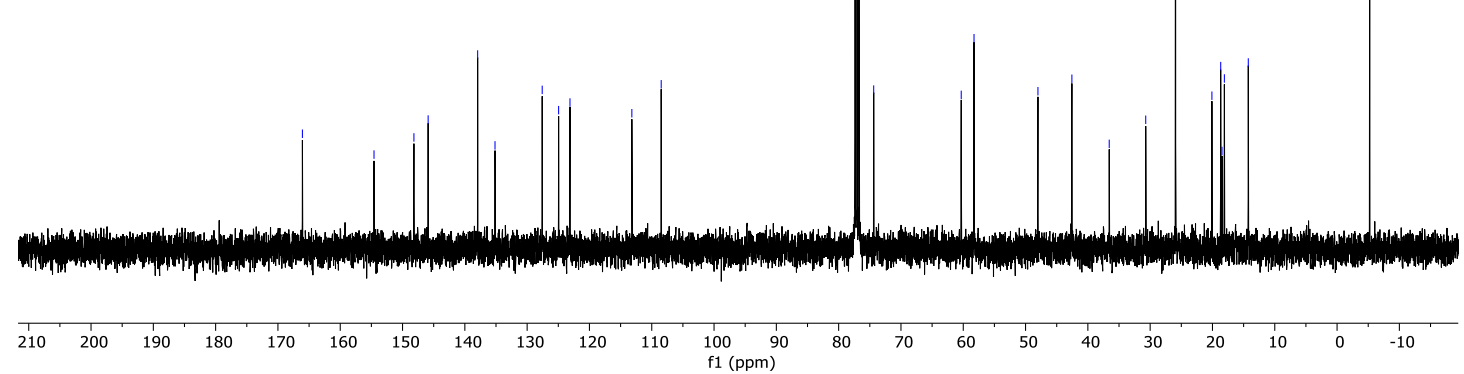




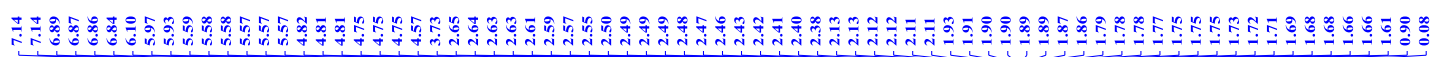

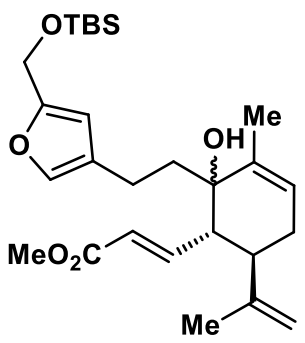

22a

( ${ }^{1} \mathrm{H}-\mathrm{NMR}, \mathrm{CDCl}_{3}, 500 \mathrm{MHz}$ )

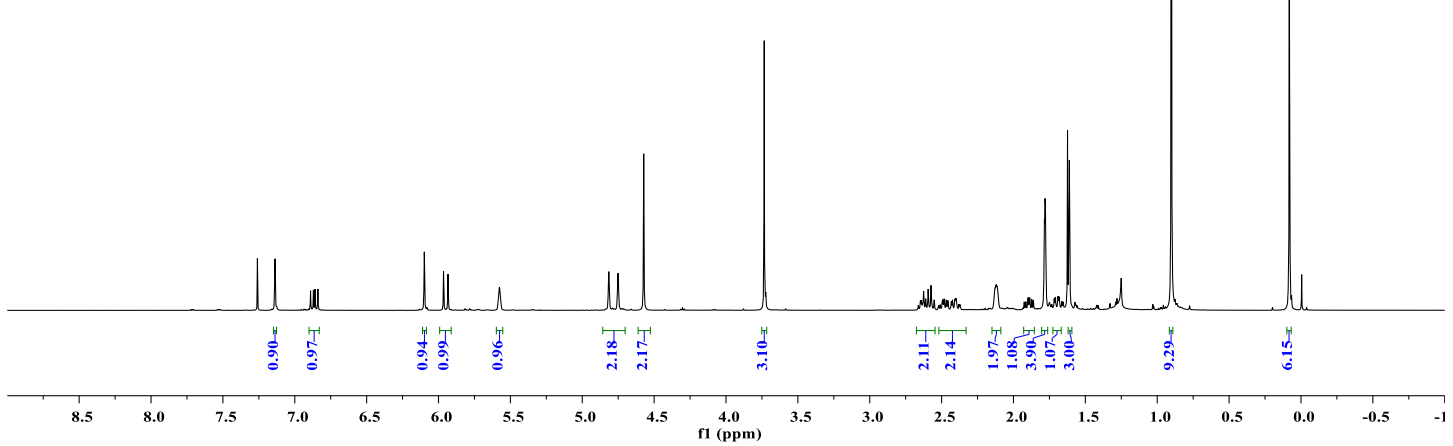

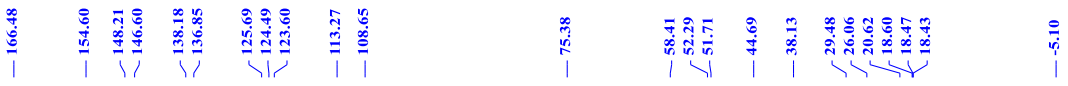

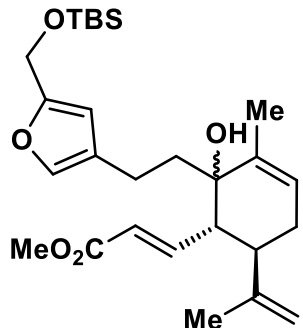

$22 a$

$\left({ }^{13} \mathrm{C}-\mathrm{NMR}, \mathrm{CDCl}_{3}, 126 \mathrm{MHz}\right)$

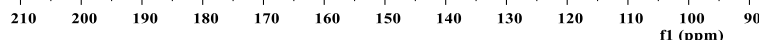




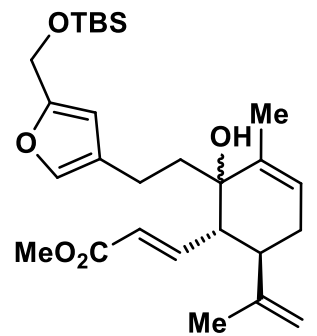

22b

$\left({ }^{1} \mathrm{H}-\mathrm{NMR}, \mathrm{CDCl}_{3}, 500 \mathrm{MHz}\right)$

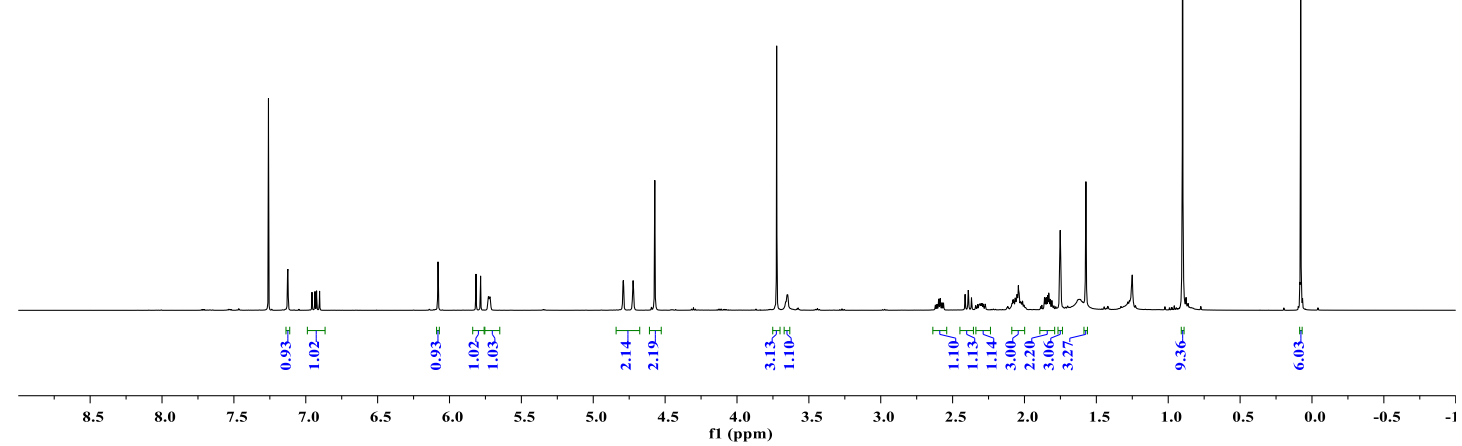

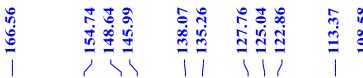

章<smiles>C=C(C)[C@H]1CC=C(C)[C@](O)(CCc2coc(CO[Sb])c2)[C@@H]1/C=C/C(C)=O</smiles>

22b

$\left({ }^{13} \mathrm{C}-\mathrm{NMR}, \mathrm{CDCl}_{3}, 126 \mathrm{MHz}\right)$

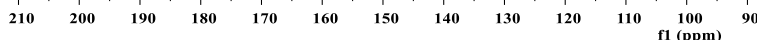




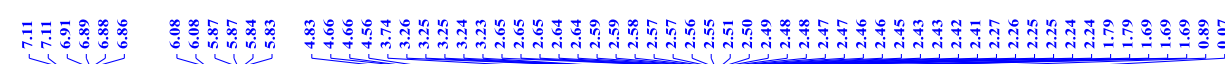<smiles>C=C(C)[C@H]1CC(=O)C([N+](=O)[O-])=C(CCc2coc(COC(C)C)c2)[C@H]1/C=C/C(C)=O</smiles>

23

$\left({ }^{1} \mathrm{H}-\mathrm{NMR}, \mathrm{CDCl}_{3}, 500 \mathrm{MHz}\right)$

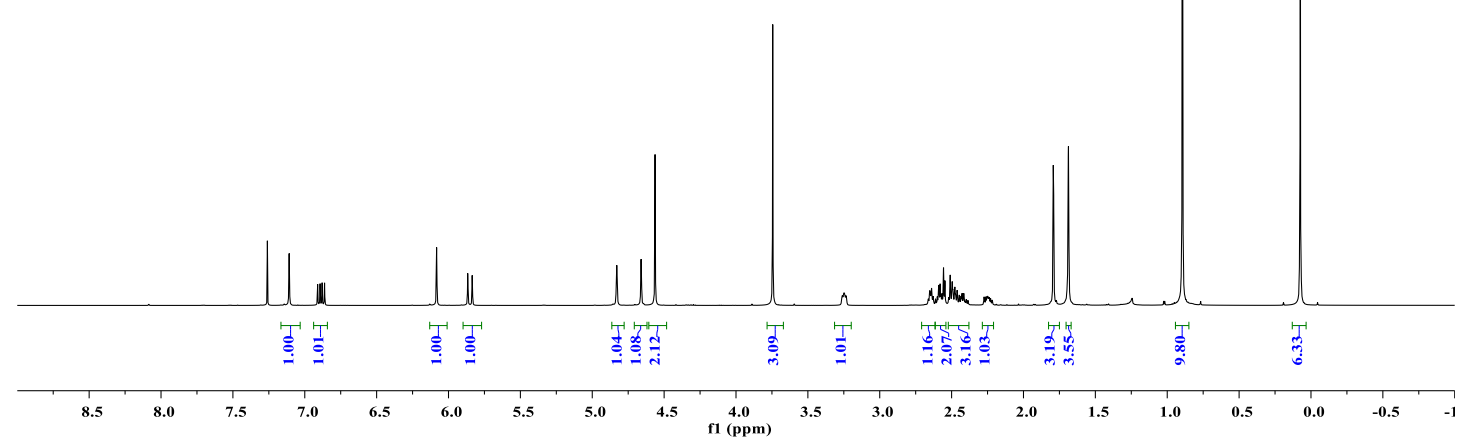

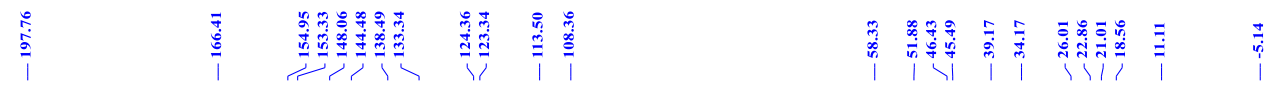

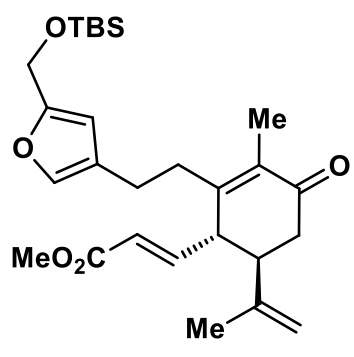

23

$\left({ }^{13} \mathrm{C}-\mathrm{NMR}, \mathrm{CDCl}_{3}, 126 \mathrm{MHz}\right)$

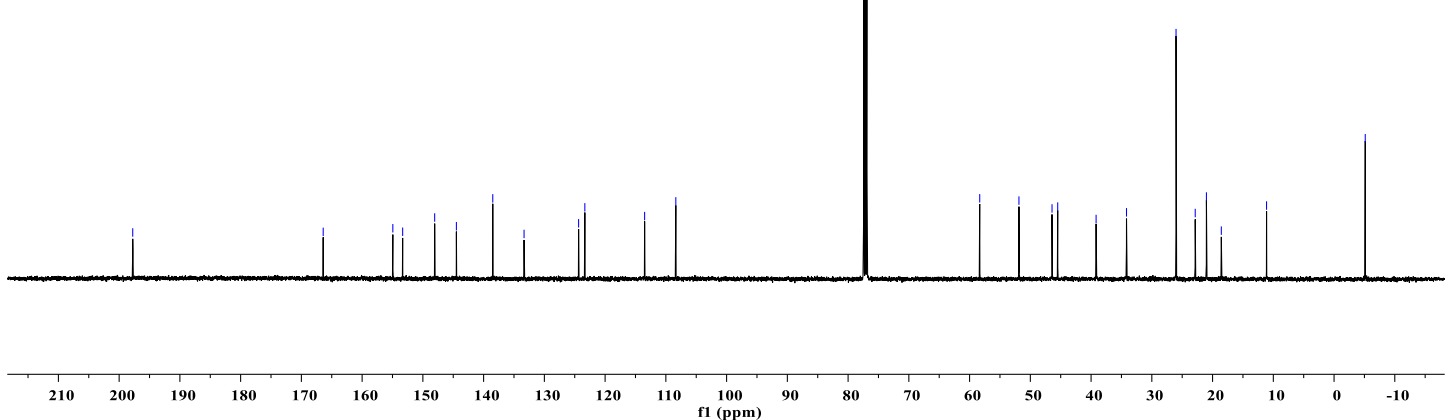




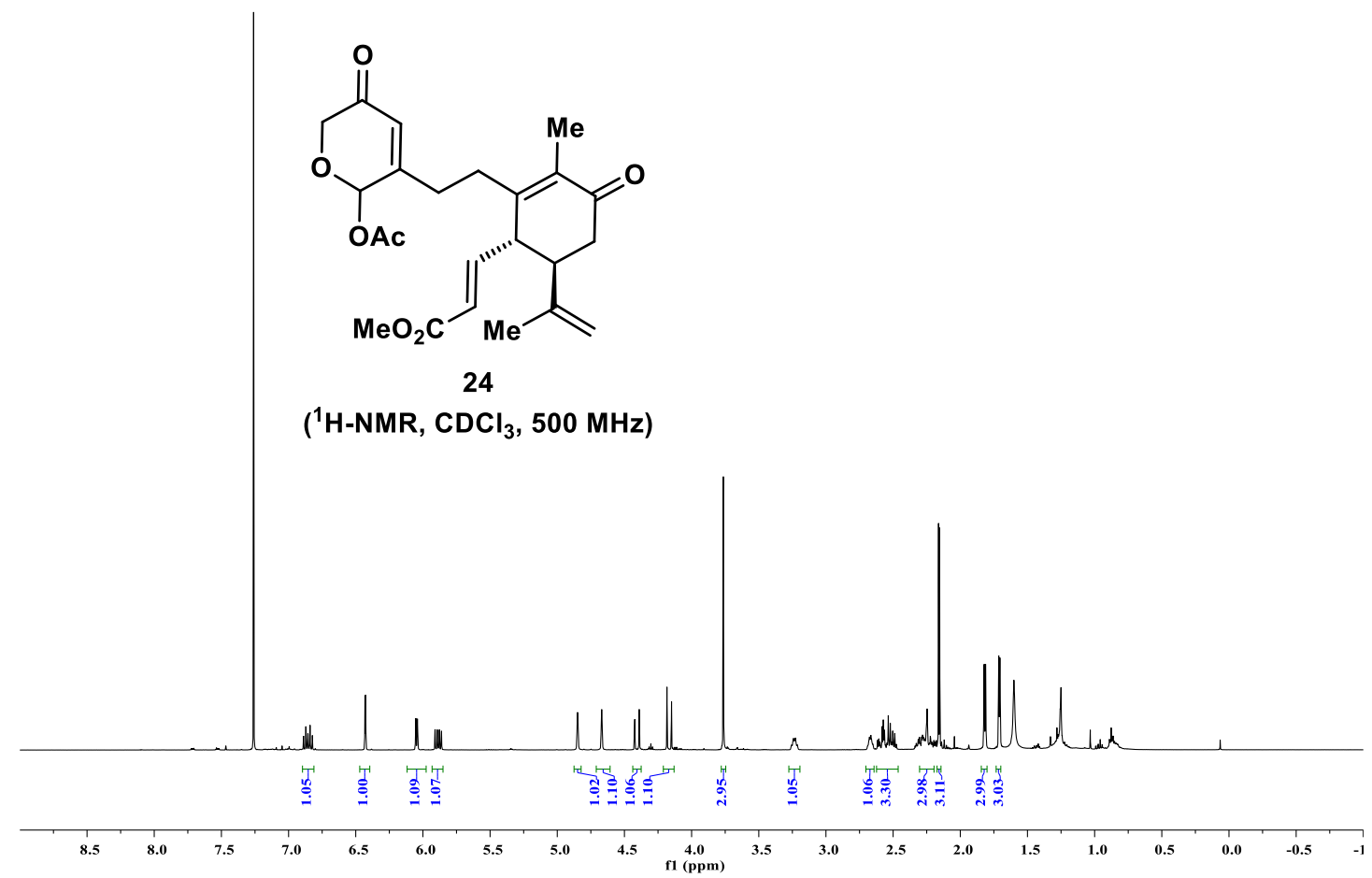

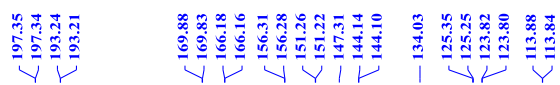

聯<smiles>C=C(C)[C@H]1CC(=O)C([N+](=O)[O-])=C(CCC2=CC(=O)COC2OC(C)=O)[C@H]1/C=C/C(C)=O</smiles>

$\left({ }^{13} \mathrm{C}-\mathrm{NMR}, \mathrm{CDCl}_{3}, 126 \mathrm{MHz}\right)$

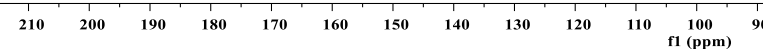




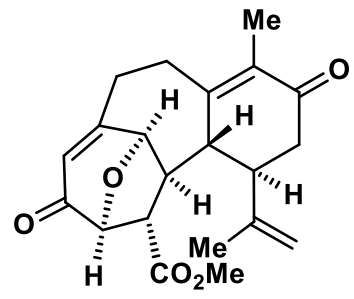

25

( ${ }^{1} \mathrm{H}-\mathrm{NMR}, \mathrm{CDCl}_{3}, 500 \mathrm{MHz}$ )

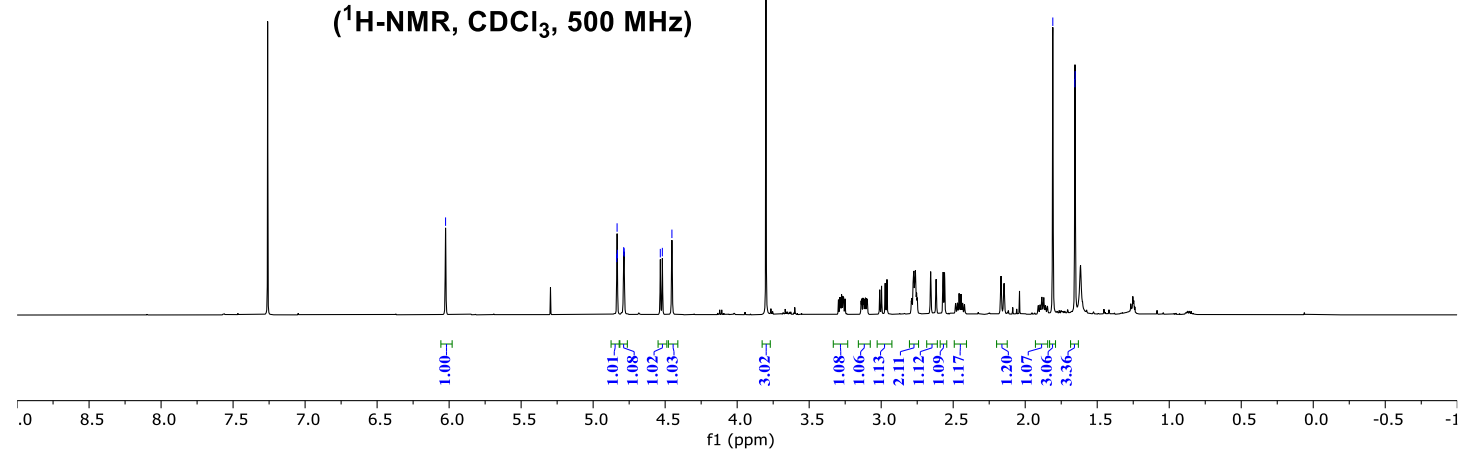

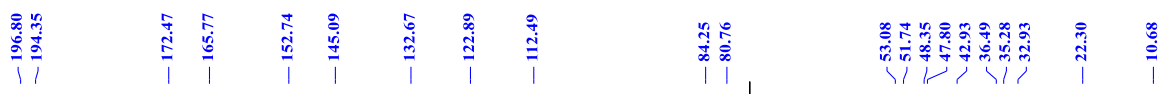

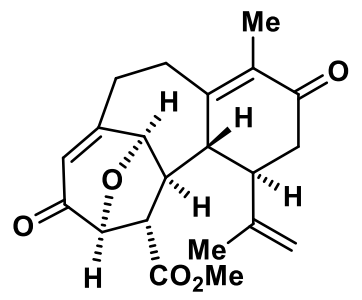

25

$\left({ }^{13} \mathrm{C}-\mathrm{NMR}, \mathrm{CDCl}_{3}, 126 \mathrm{MHz}\right)$

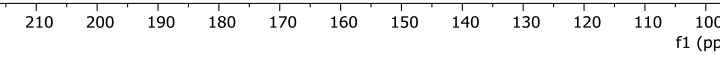




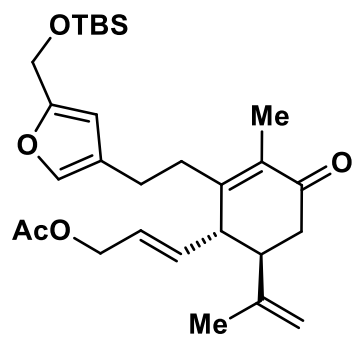

26

(' ${ }^{1} \mathrm{H}-\mathrm{NMR}, \mathrm{CDCl}_{3}, 500 \mathrm{MHz}$ )
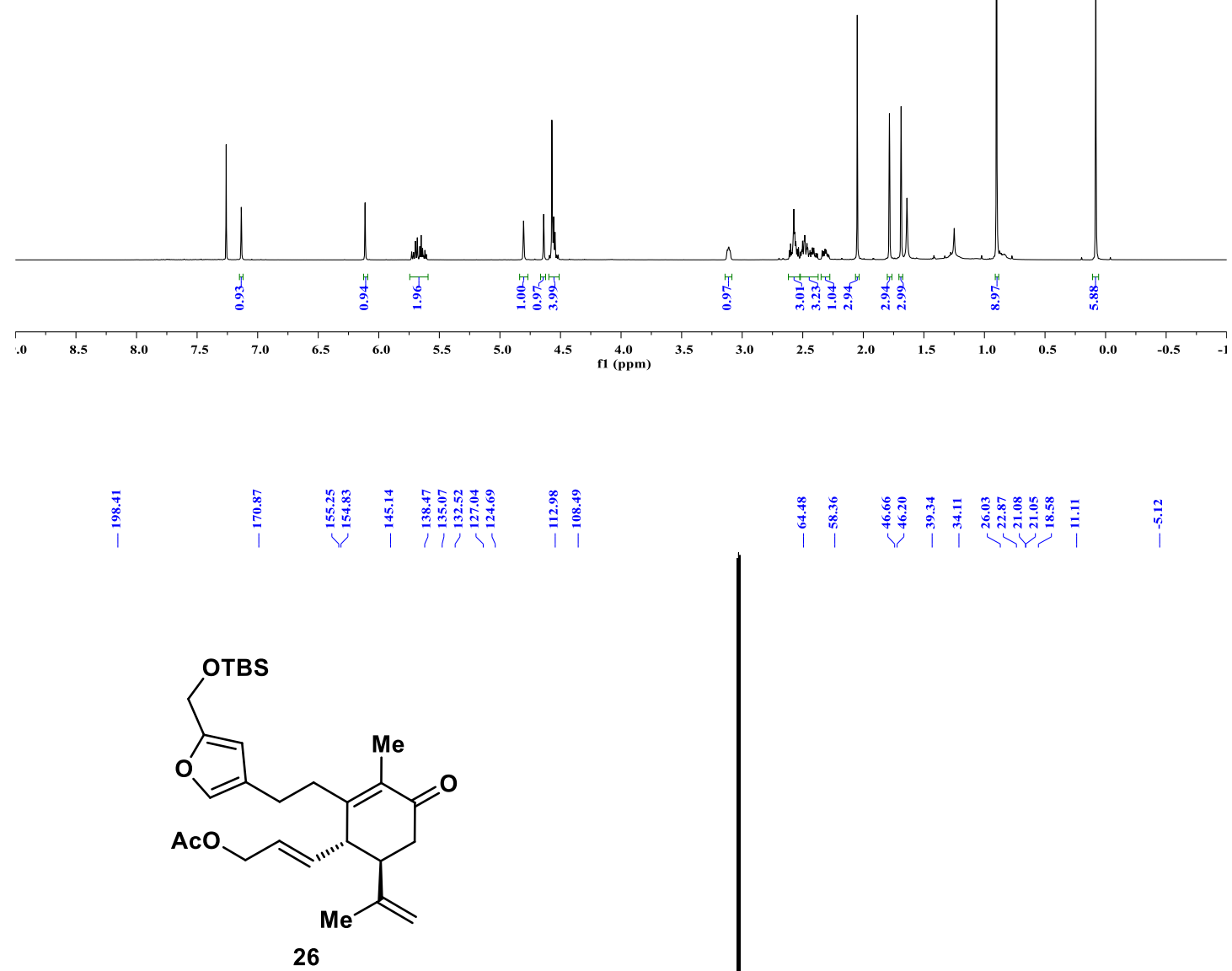

$\left({ }^{13} \mathrm{C}-\mathrm{NMR}, \mathrm{CDCl}_{3}, 126 \mathrm{MHz}\right)$

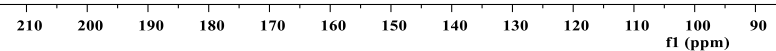

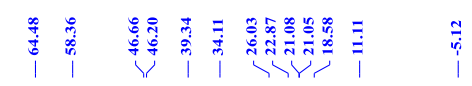

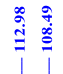

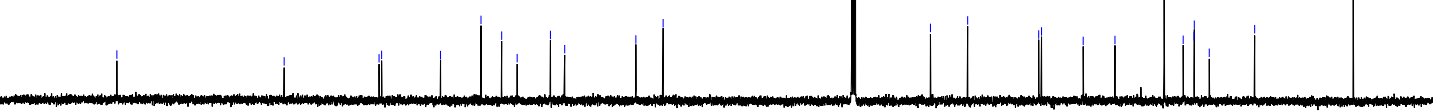




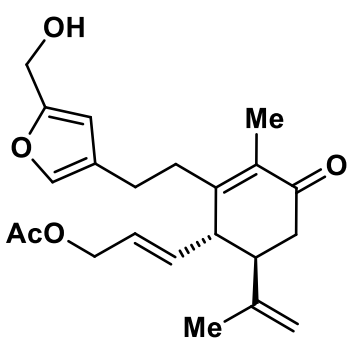

27

$\left({ }^{1} \mathrm{H}-\mathrm{NMR}, \mathrm{CDCl}_{3}, 500 \mathrm{MHz}\right)$

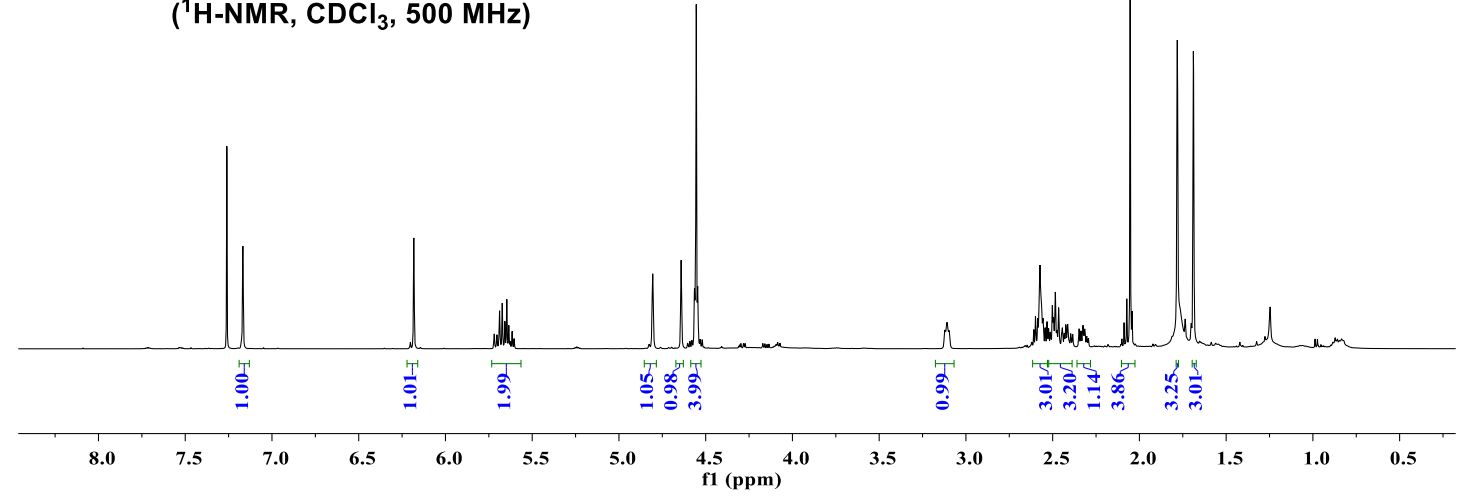

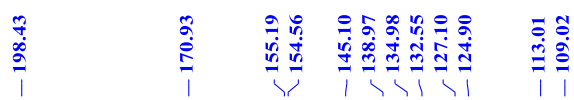

点<smiles>C=C(C)[C@H]1CC(=O)C(C)=C(CCc2coc(CO)c2)[C@H]1/C=C/COC(C)=O</smiles>

$\left({ }^{13} \mathrm{C}-\mathrm{NMR}, \mathrm{CDCl}_{3}, 126 \mathrm{MHz}\right)$ 


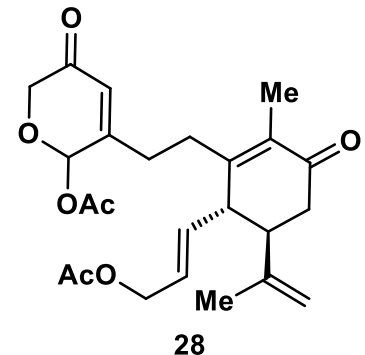

( ${ }^{1} \mathrm{H}-\mathrm{NMR}, \mathrm{CDCl}_{3}, 500 \mathrm{MHz}$ )

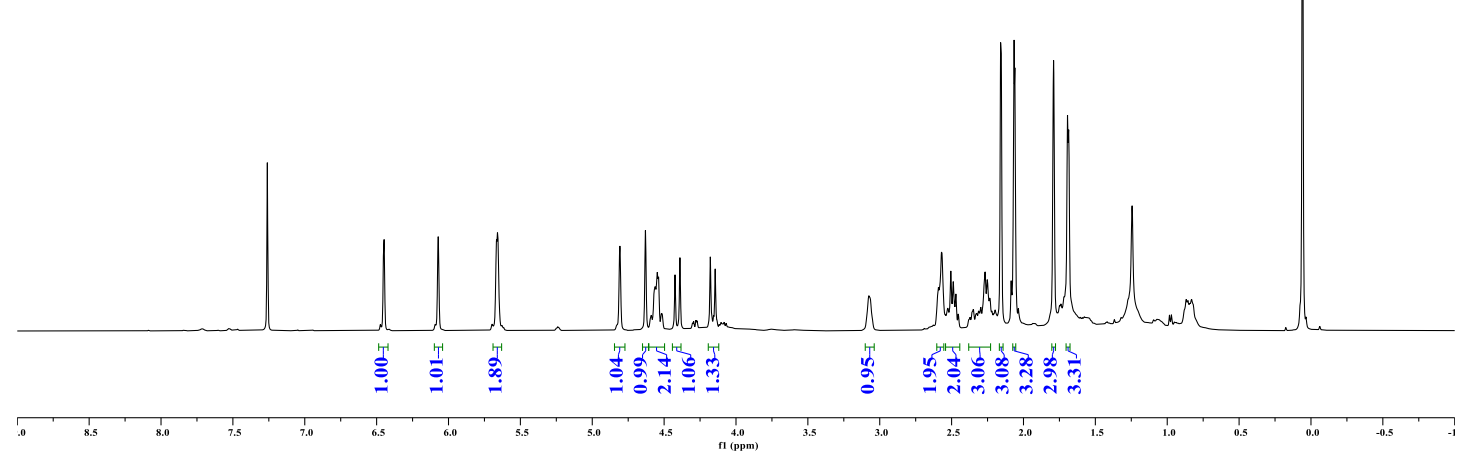

स.

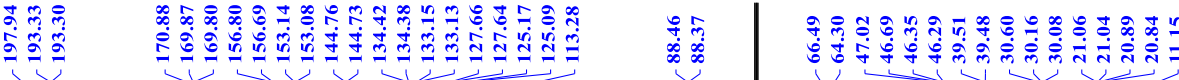<smiles>C=C(C)[C@H]1CC(=O)C(C)=C(CCC2=CC(=O)COC2OC(C)=O)[C@H]1/C=C/COC(C)=O</smiles>

28

$\left({ }^{13} \mathrm{C}-\mathrm{NMR}, \mathrm{CDCl}_{3}, 126 \mathrm{MHz}\right)$

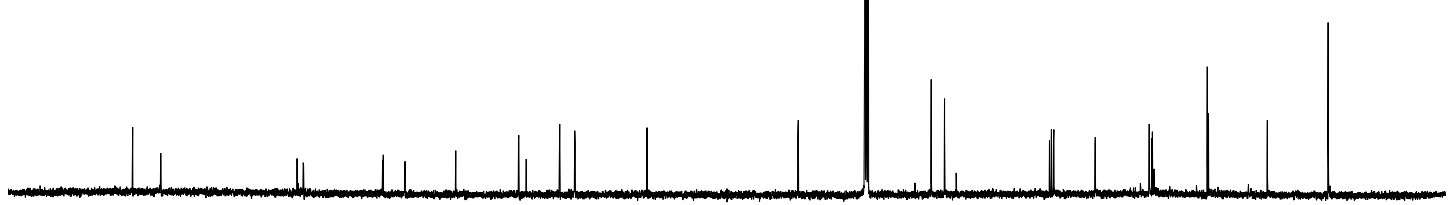

$\begin{array}{lllllllllllllllllllllll}210 & 200 & 190 & 180 & 170 & 160 & 150 & 140 & 130 & 120 & 110 & 100 & 90 & 80 & 70 & 60 & 50 & 40 & 30 & 20 & 10 & 0 & -10\end{array}$ 


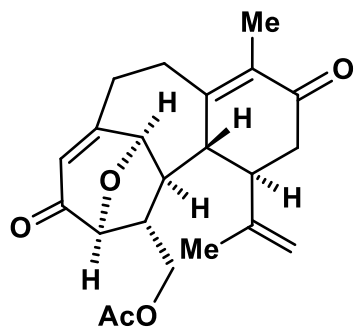

29

( $\left.{ }^{1} \mathrm{H}-\mathrm{NMR}, \mathrm{CDCl}_{3}, 500 \mathrm{MHz}\right)$

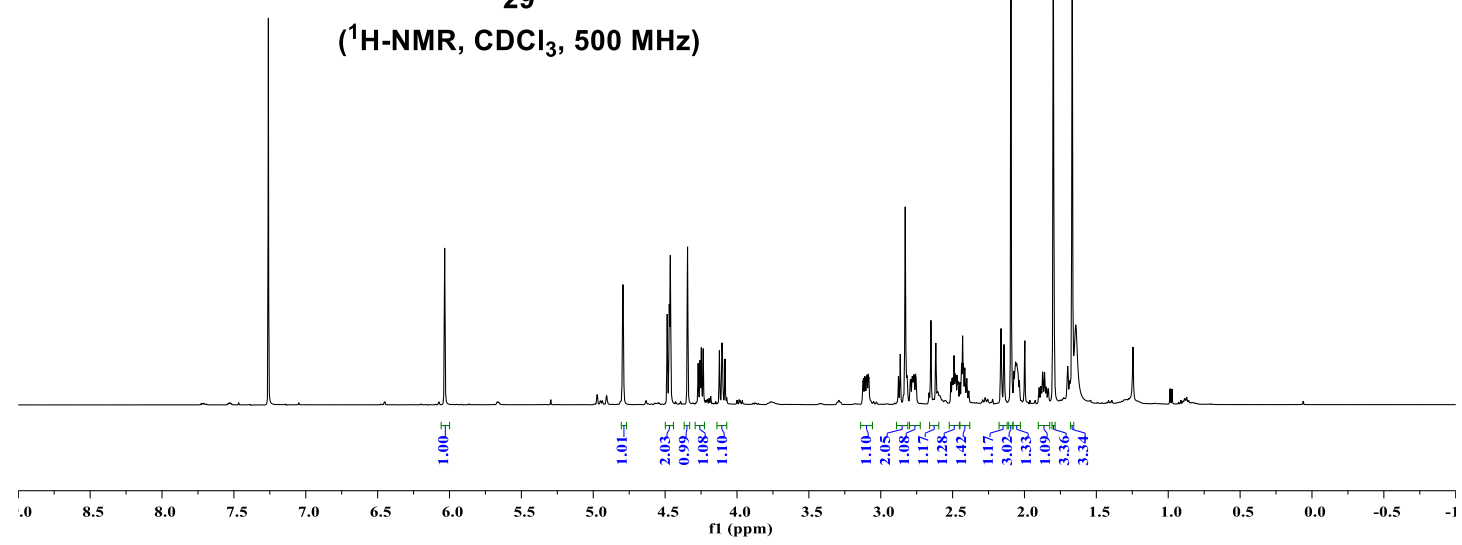

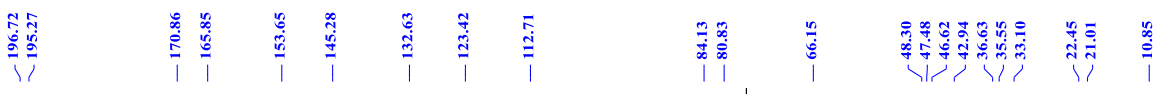

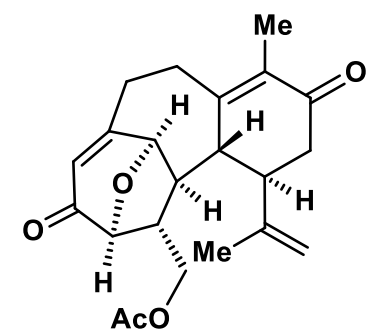

29

$\left({ }^{13} \mathrm{C}-\mathrm{NMR}, \mathrm{CDCl}_{3}, 126 \mathrm{MHz}\right)$

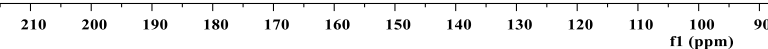




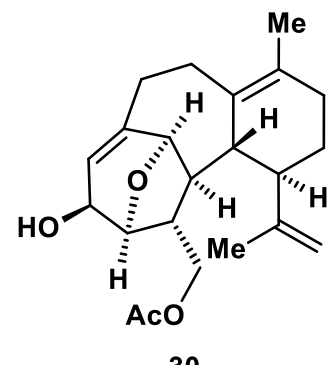

(' $\left.{ }^{1} \mathrm{H}-\mathrm{NMR}, \mathrm{CDCl}_{3}, 500 \mathrm{MHz}\right)$

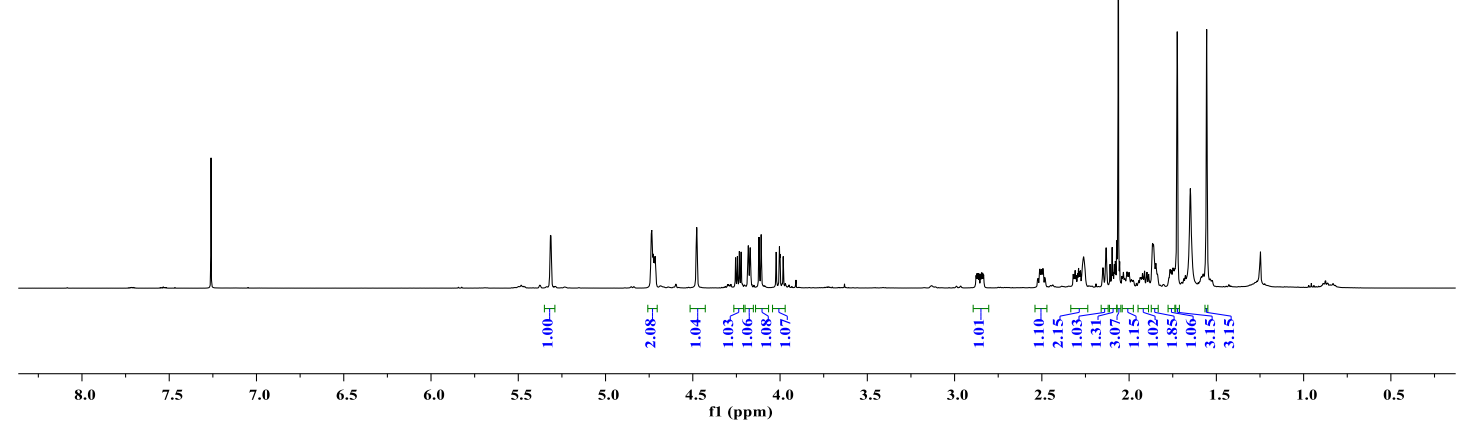

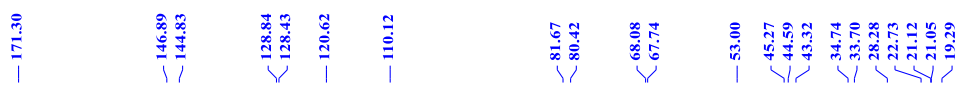

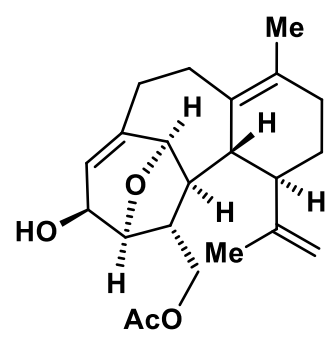

30

$\left({ }^{13} \mathrm{C}-\mathrm{NMR}, \mathrm{CDCl}_{3}, 126 \mathrm{MHz}\right)$

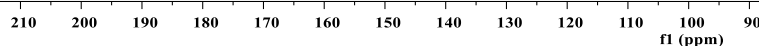




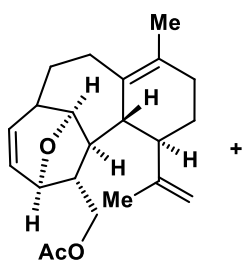

31

('H-NMR, $\mathrm{CDCl}_{3}, 500 \mathrm{MHz}$ )

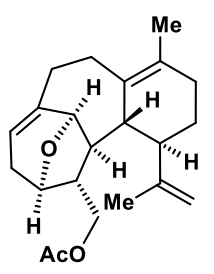

32

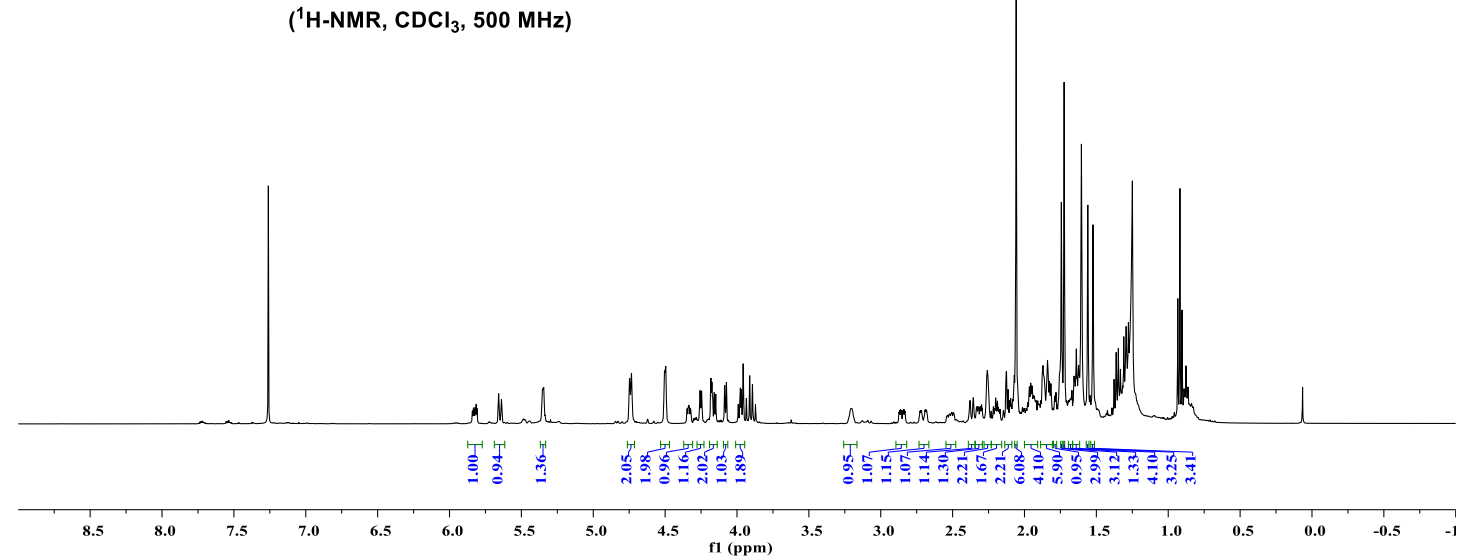

竞

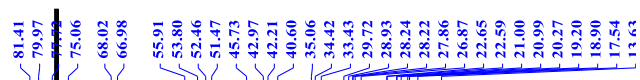
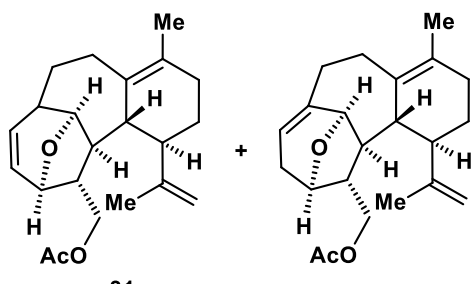

$\left({ }^{13} \mathrm{C}\right.$-NMR, $\mathrm{CDCl}_{3}, 126 \mathrm{MHz}$ )

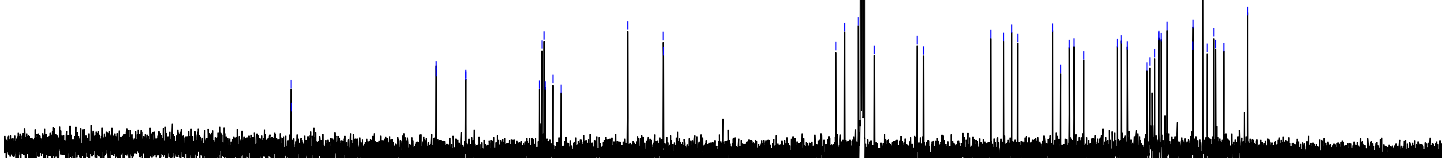

$\begin{array}{llllllllllll}200 & 190 & 180 & 170 & 160 & 150 & 140 & 130 & 120 & 110 & \underset{f 1}{100}(\mathrm{ppm}) & 90\end{array}$ 


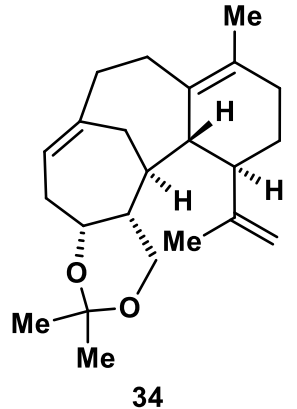

( $\left.{ }^{1} \mathrm{H}-\mathrm{NMR}, \mathrm{CDCl}_{3}, 500 \mathrm{MHz}\right)$
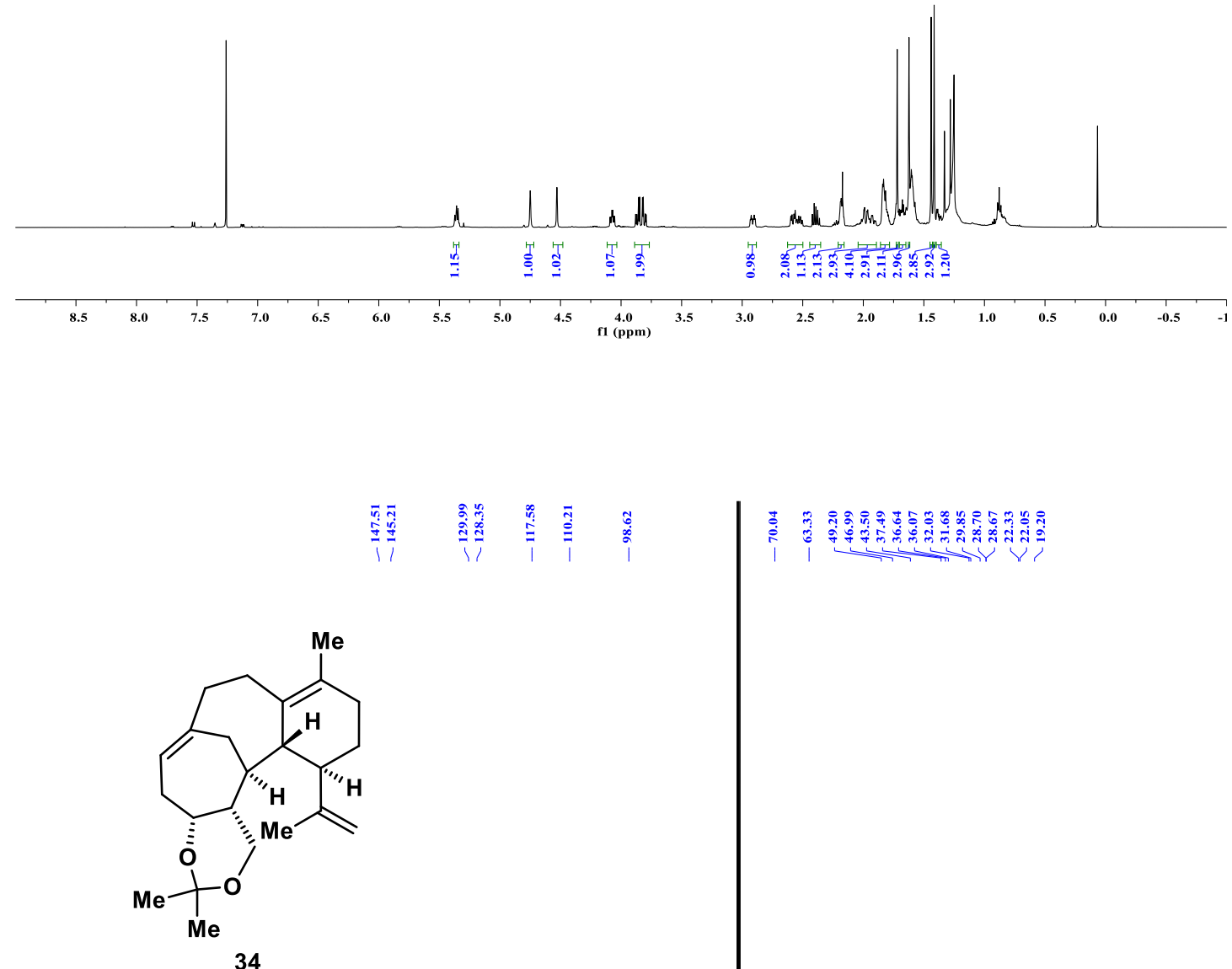

$\left({ }^{13} \mathrm{C}-\mathrm{NMR}, \mathrm{CDCl}_{3}, 126 \mathrm{MHz}\right)$

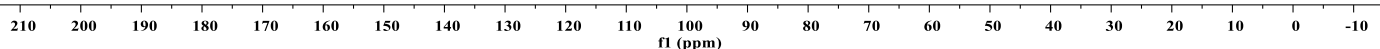




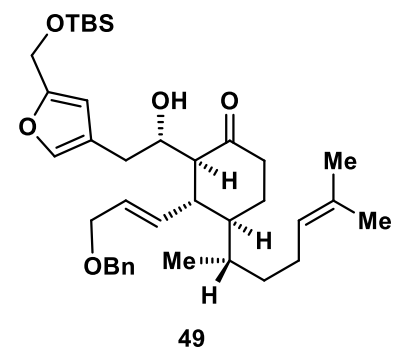

( ${ }^{1} \mathrm{H}-\mathrm{NMR}, \mathrm{CDCl}_{3}, 500 \mathrm{MHz}$ )
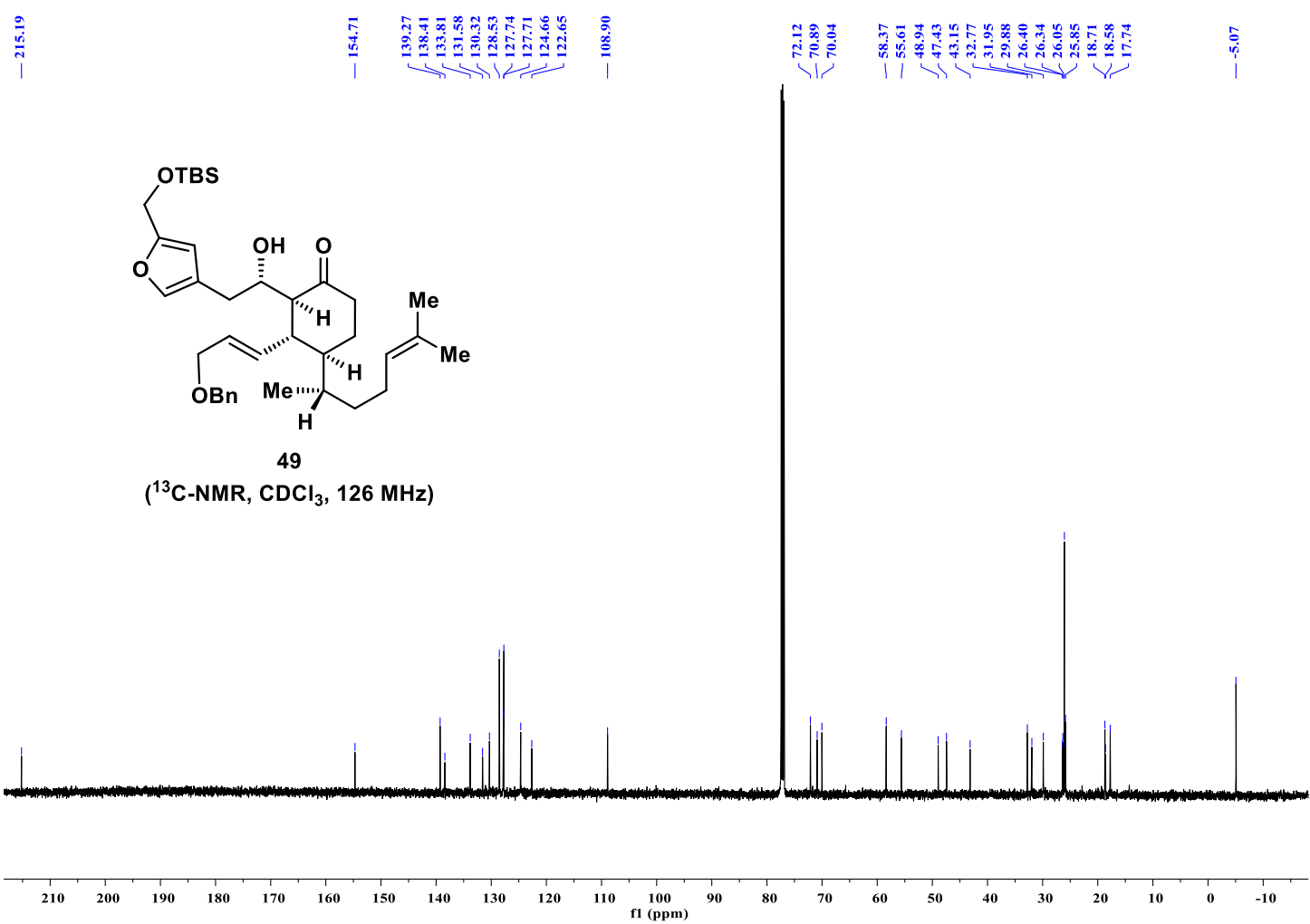


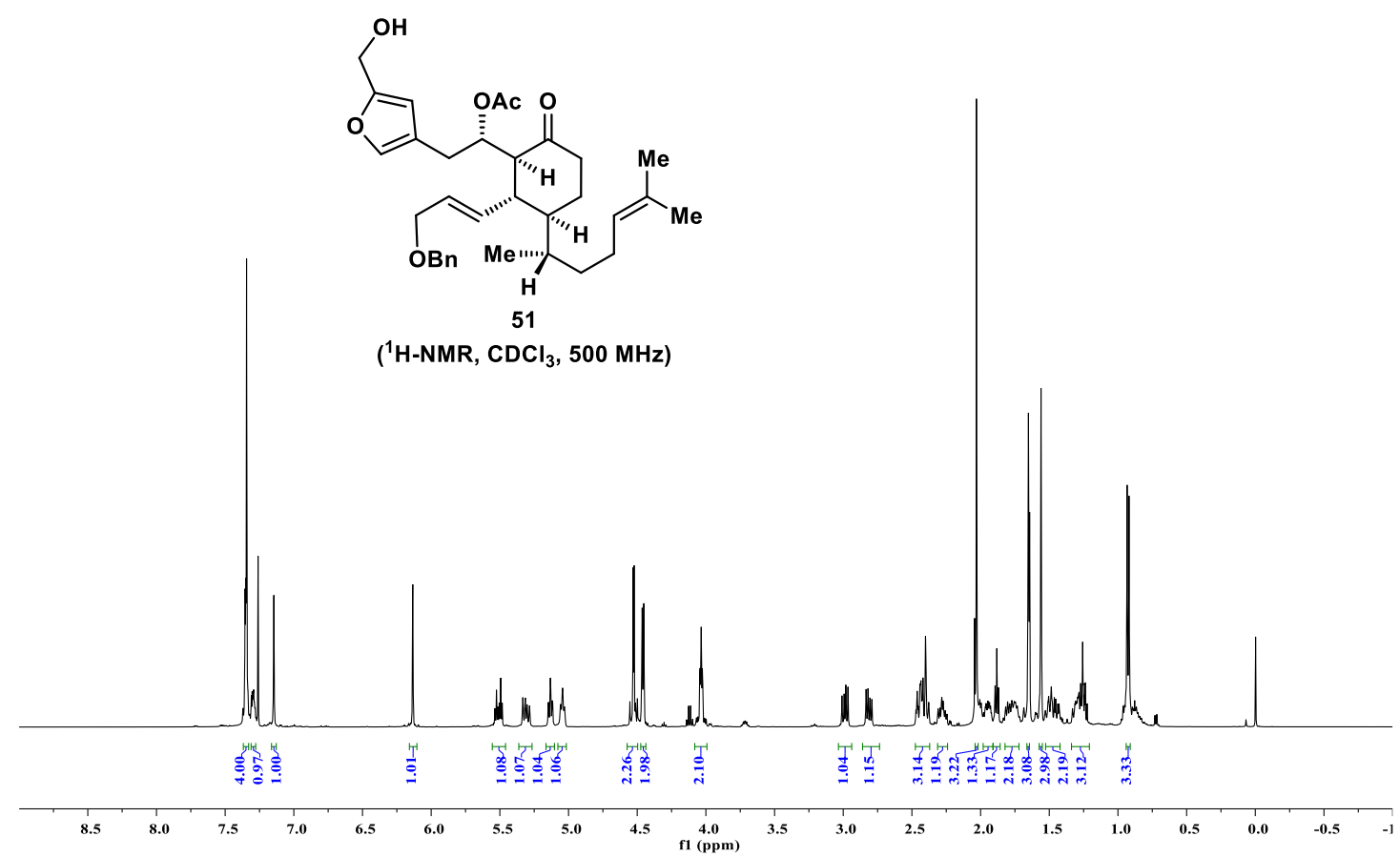

等

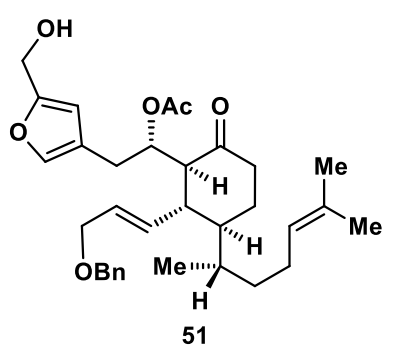

$\left({ }^{13} \mathrm{C}-\mathrm{NMR}, \mathrm{CDCl}_{3}, 126 \mathrm{MHz}\right)$

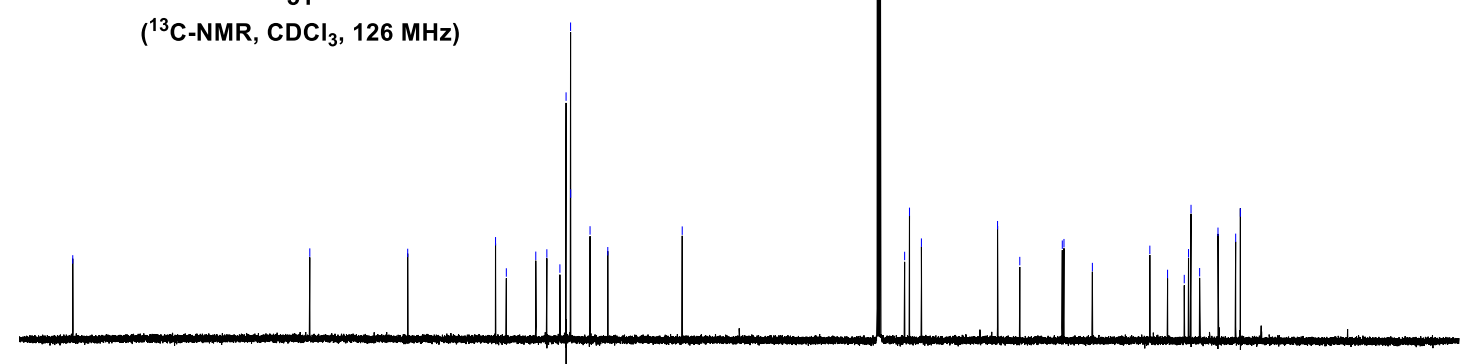

$\begin{array}{lllllllllllllllllllllllll}210 & 200 & 190 & 180 & 170 & 160 & 150 & 140 & 130 & 120 & 110 & \underset{f 1}{100}(\mathrm{ppm}) & 90 & 80 & 70 & 60 & 50 & 40 & 30 & 20 & 10 & 0 & -10\end{array}$ 


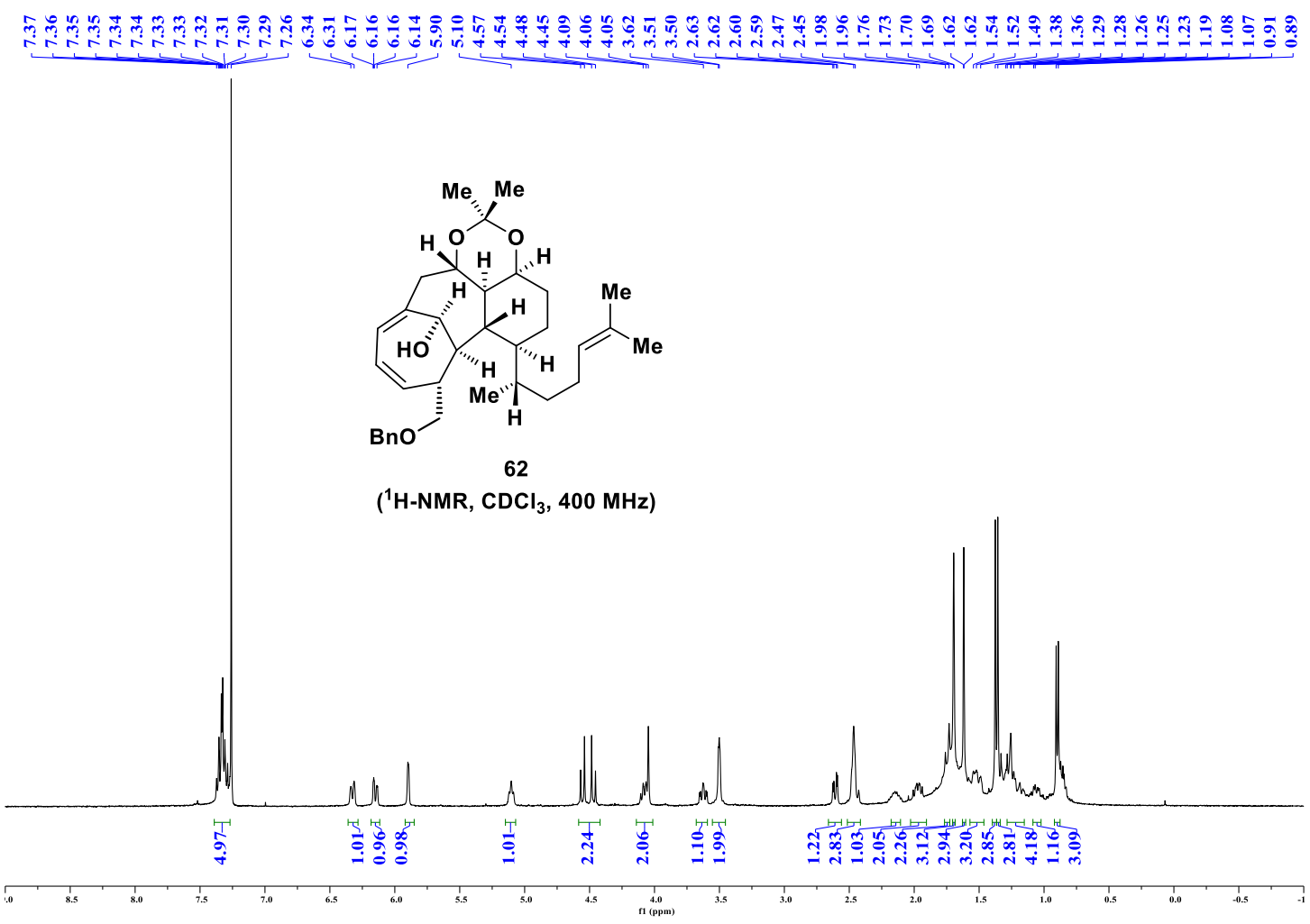

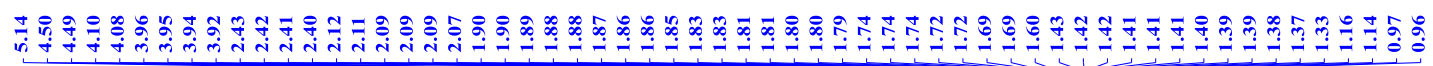

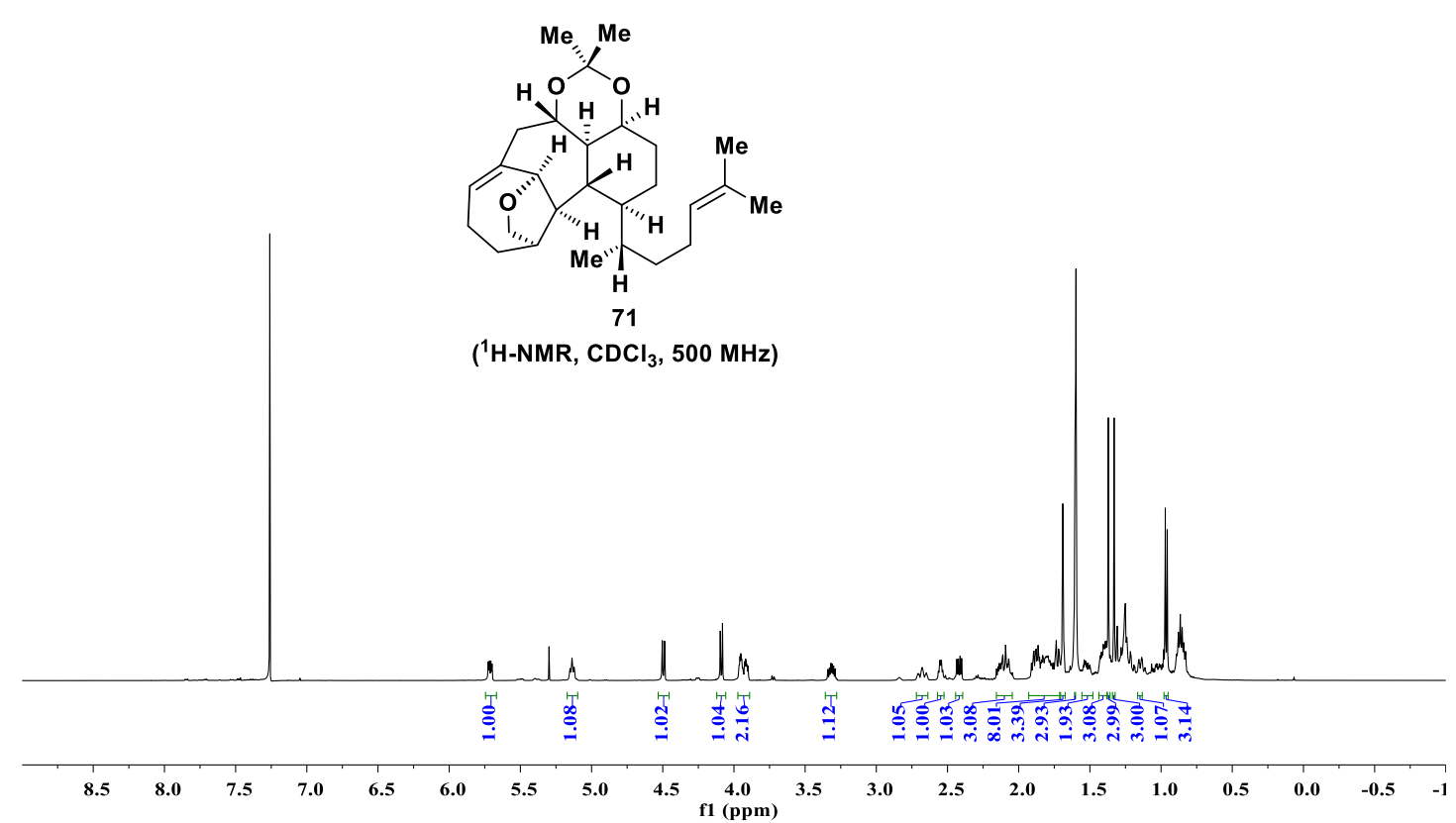




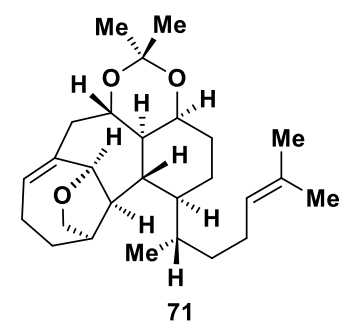

TBDPSOH

( ${ }^{1} \mathrm{H}-\mathrm{NMR}, \mathrm{CDCl}_{3}, 500 \mathrm{MHz}$ )

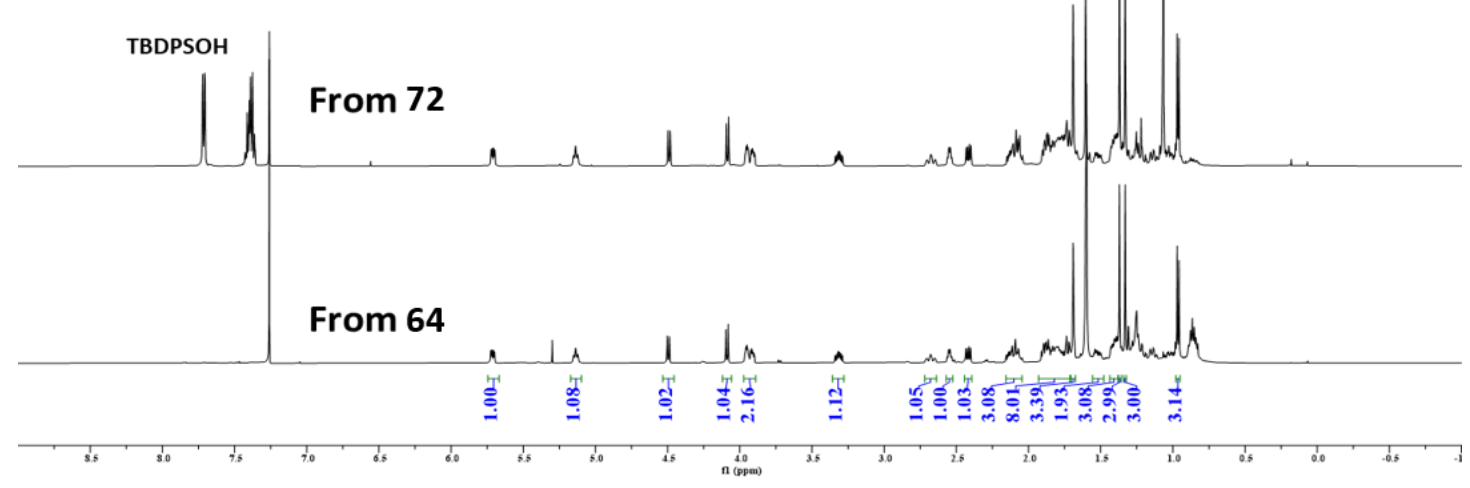

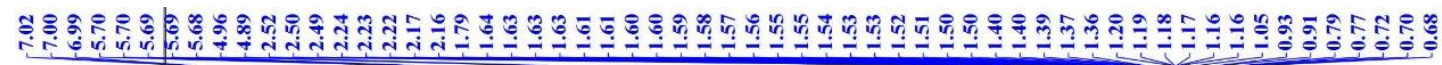

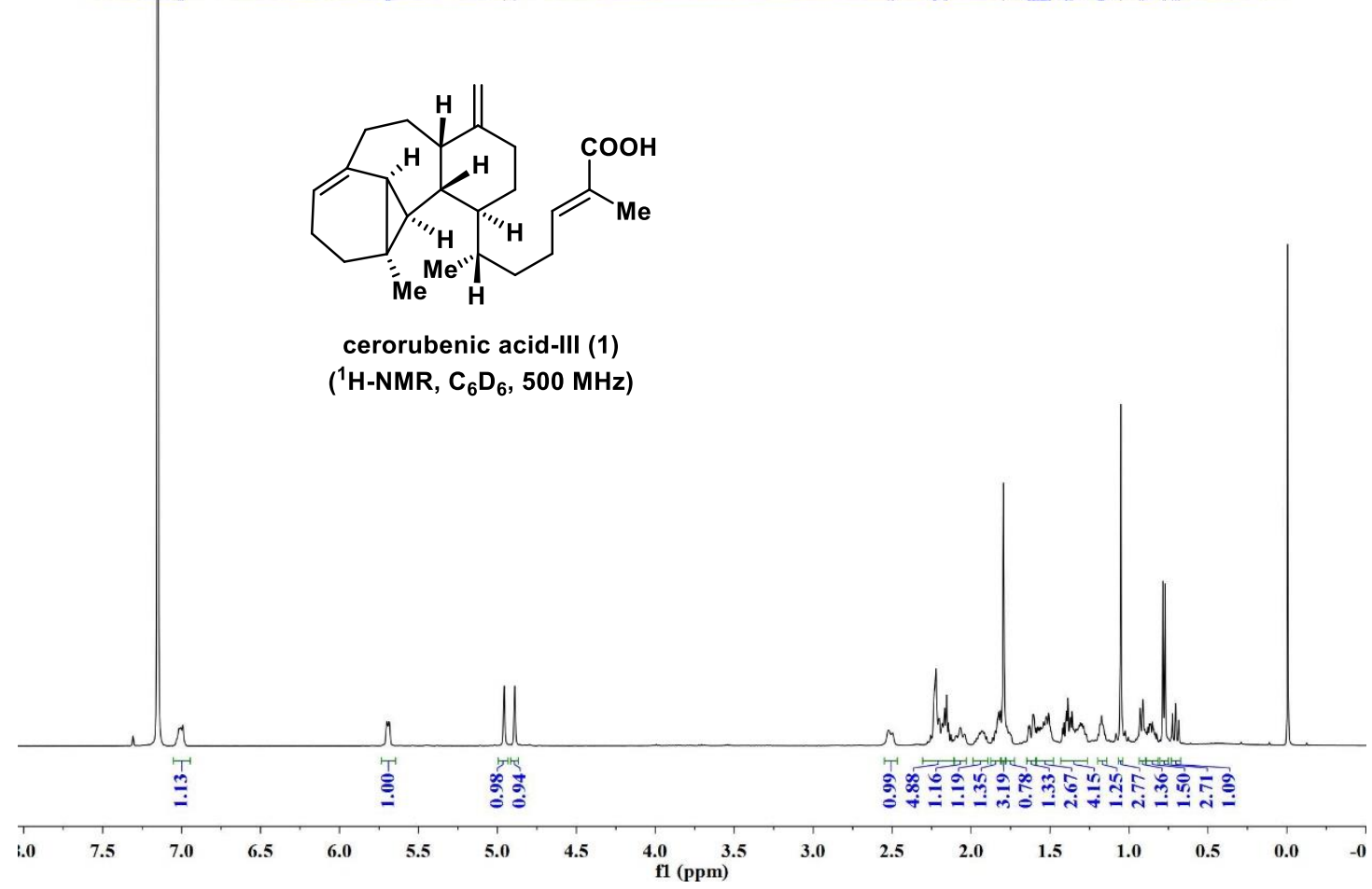




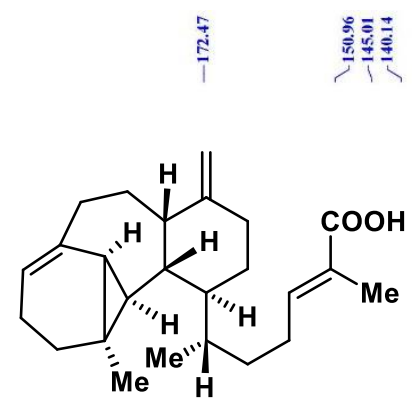

cerorubenic acid-III (1)

$\left({ }^{13} \mathrm{C}\right.$-NMR, $\left.\mathrm{C}_{6} \mathrm{D}_{6}, 126 \mathrm{MHz}\right)$
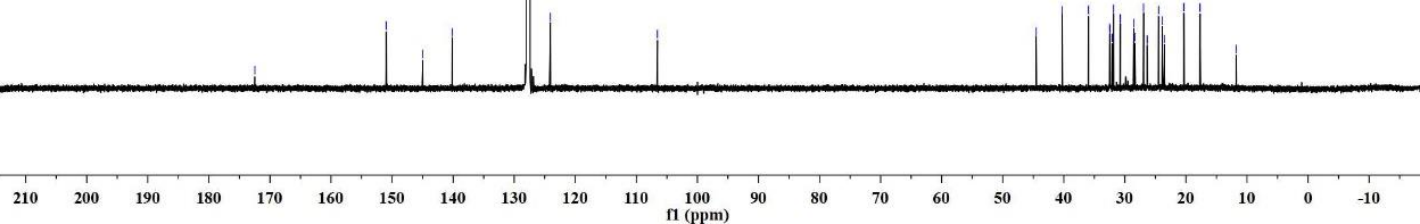

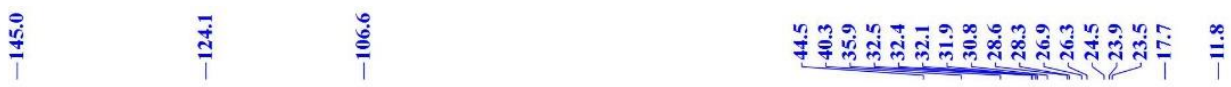

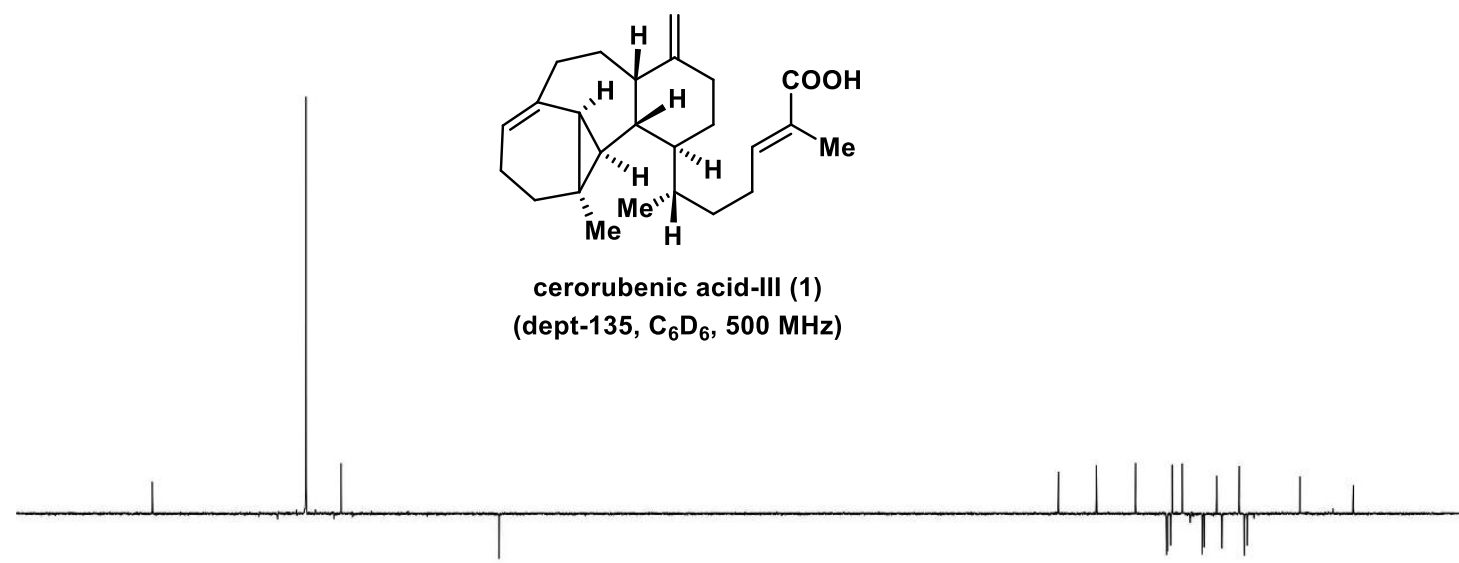

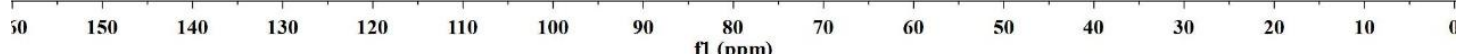




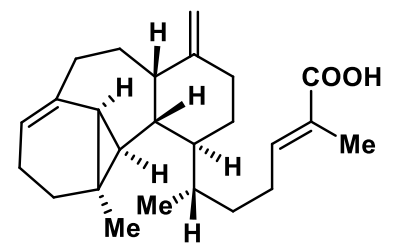

cerorubenic acid-III (1)

( ${ }^{1} \mathrm{H}-\mathrm{NMR}, \mathrm{CD}_{2} \mathrm{Cl}_{2}, 500 \mathrm{MHz}$ )

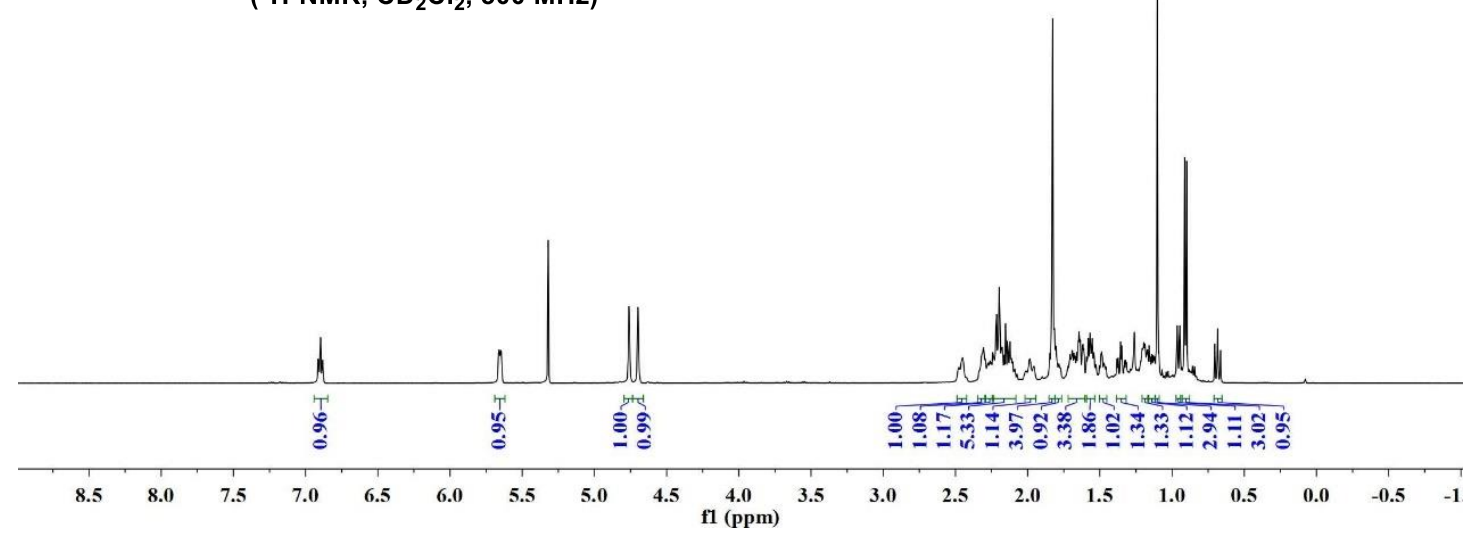

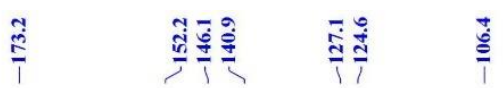
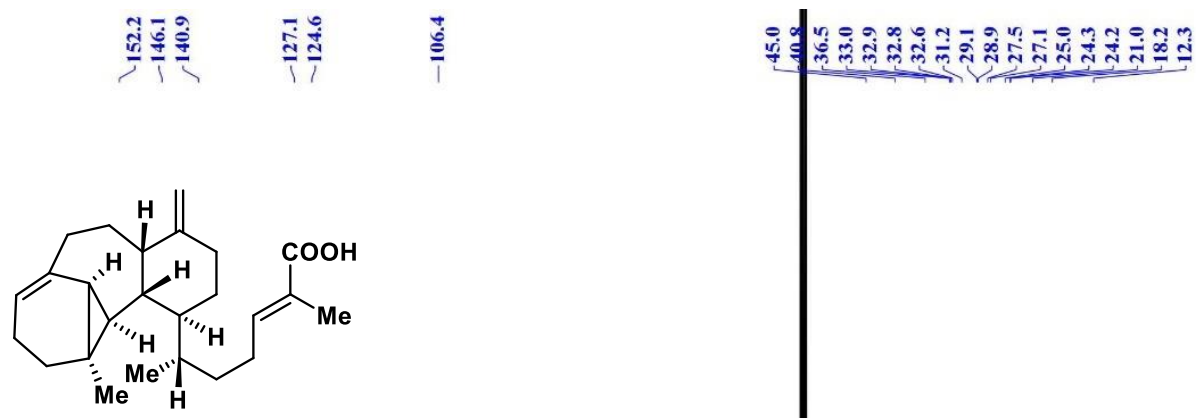

cerorubenic acid-III (1)

$\left({ }^{13} \mathrm{C}-\mathrm{NMR}, \mathrm{CD}_{2} \mathrm{Cl}_{2}, 126 \mathrm{MHz}\right)$

$\begin{array}{llllllllll}190 & 180 & 170 & 160 & 150 & 140 & 130 & 120 & 110 & \begin{array}{c}100 \\ \text { f1 (ppm) }\end{array}\end{array}$ 


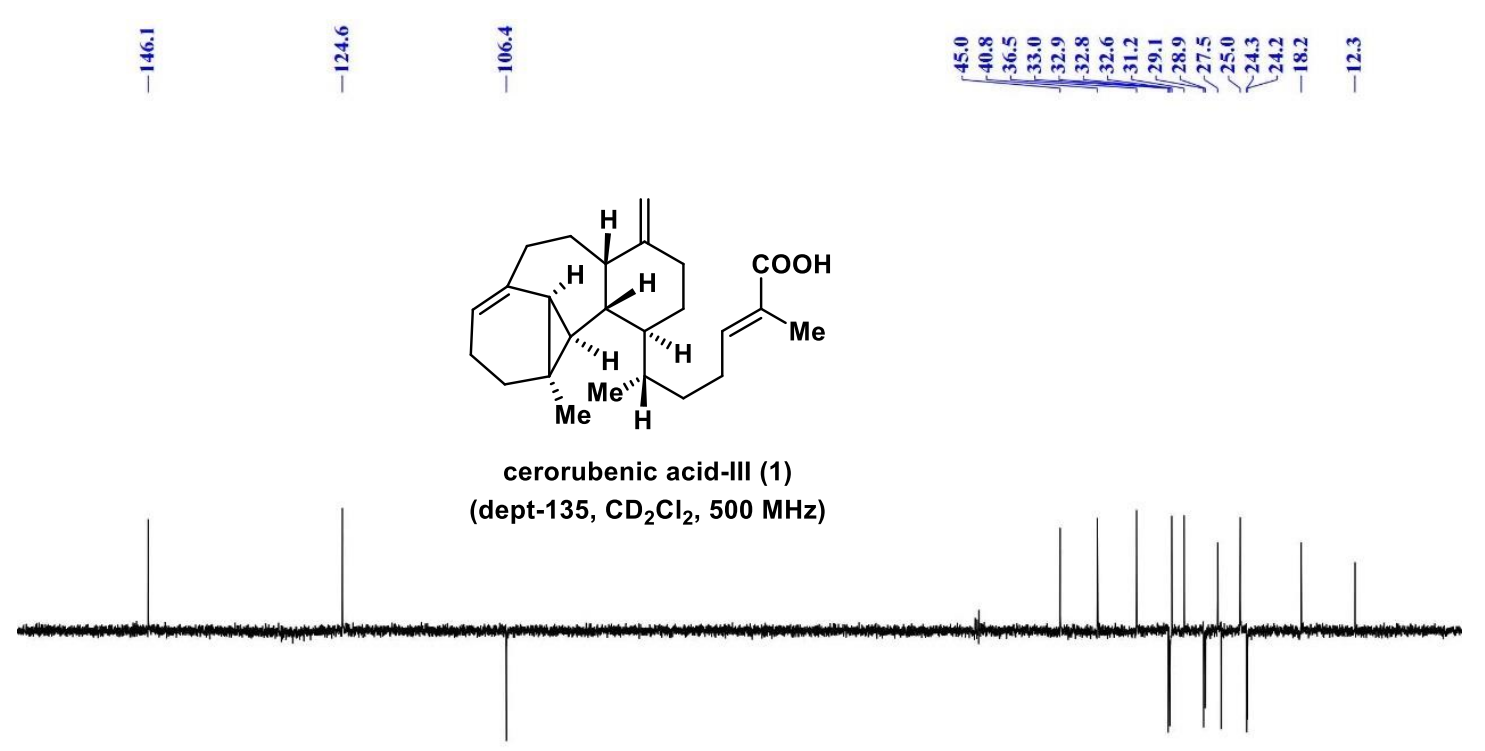

\begin{tabular}{|c|c|c|c|c|c|c|c|c|c|c|c|c|c|c|c|}
\hline 60 & 150 & 140 & 130 & 120 & 110 & 100 & 90 & $\begin{array}{c}80 \\
\text { f1 (ppm) }\end{array}$ & 70 & 60 & 50 & 40 & 30 & 20 & 10 \\
\hline
\end{tabular}

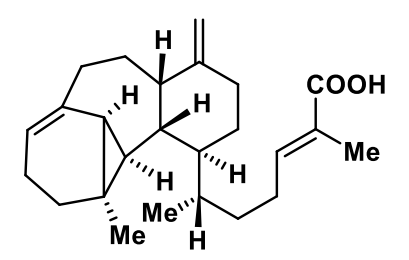

cerorubenic acid-III (1)

$\left({ }^{1} \mathrm{H}-{ }^{1} \mathrm{H}\right.$ COSY, $\left.\mathrm{CD}_{2} \mathrm{Cl}_{2}, 500 \mathrm{MHz}\right)$

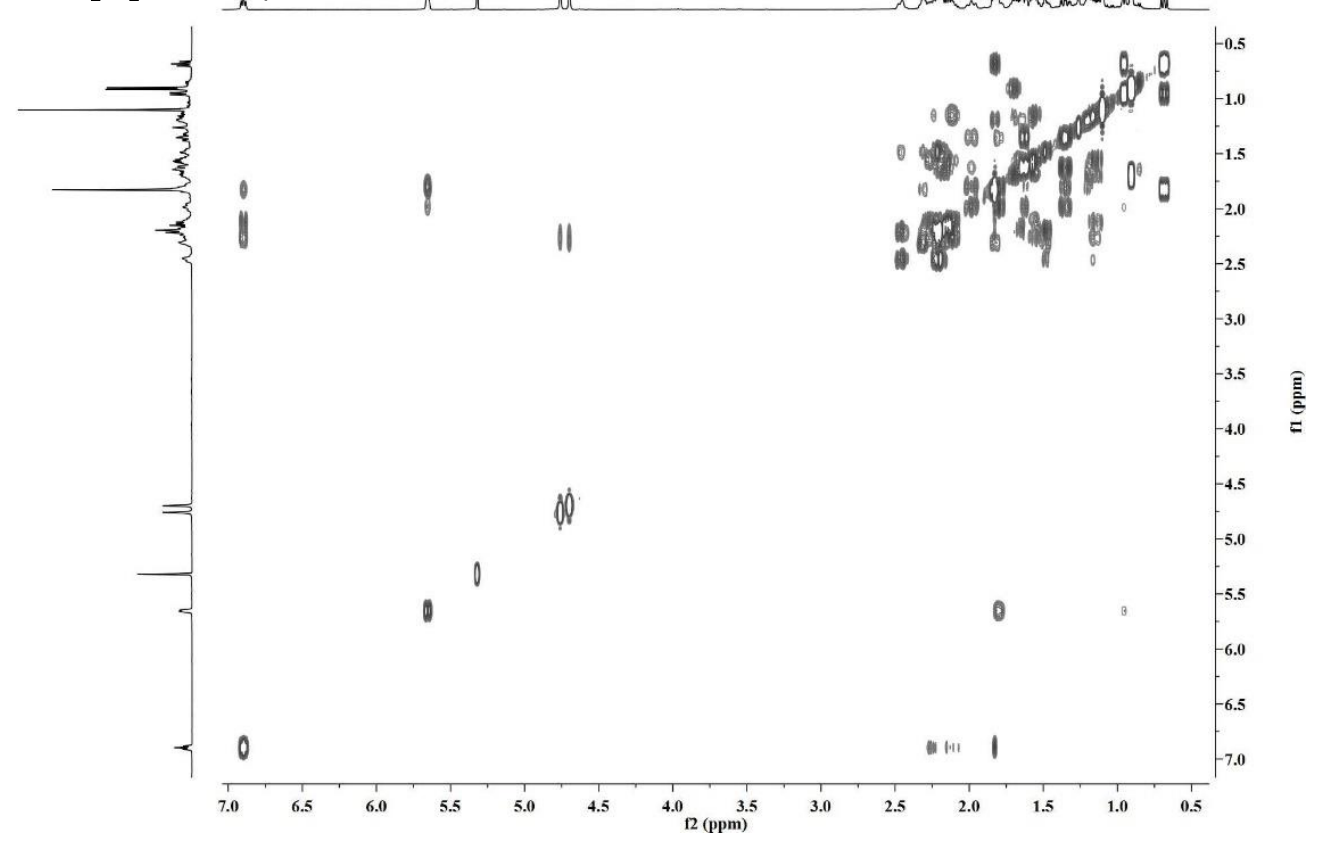




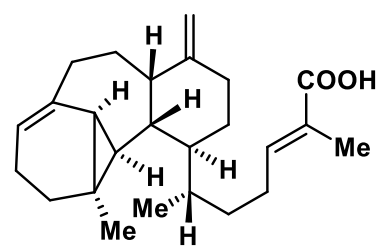

cerorubenic acid-III (1) (HSQC, $\mathrm{CD}_{2} \mathrm{Cl}_{2}, 500 \mathrm{MHz}$ )
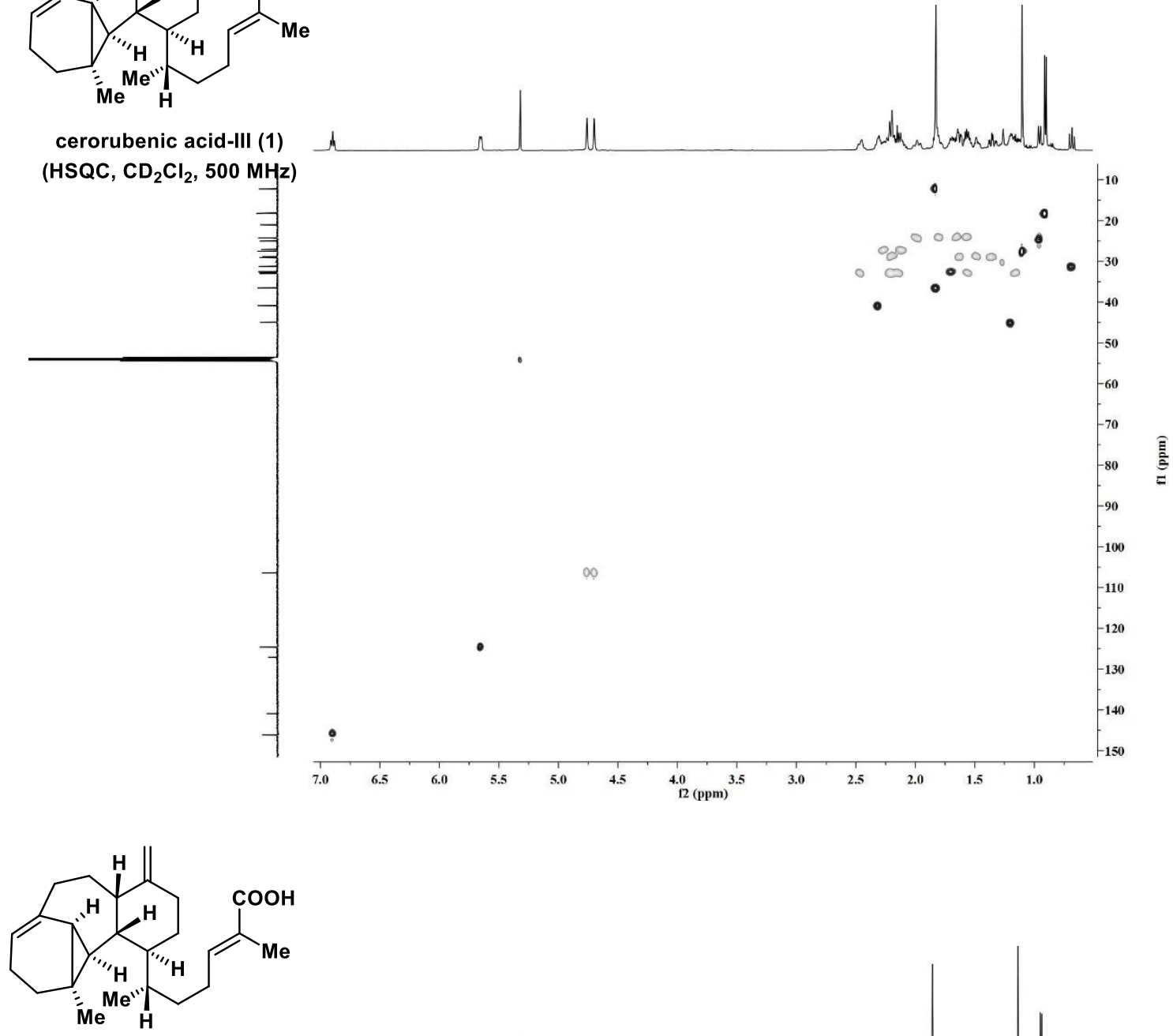

cerorubenic acid-III (1) (HMBC, $\mathrm{CD}_{2} \mathrm{Cl}_{2}, 500 \mathrm{MHz}$ )

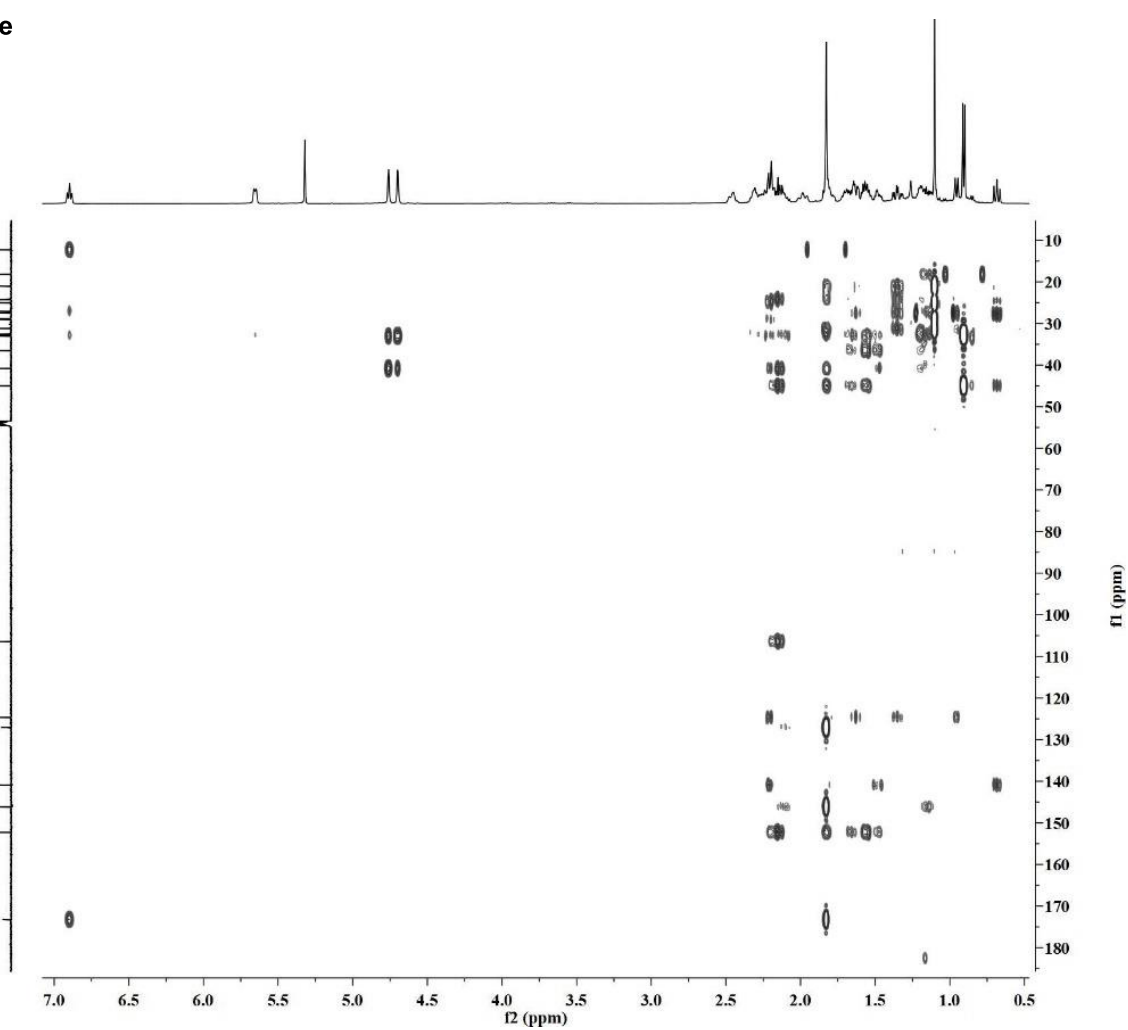




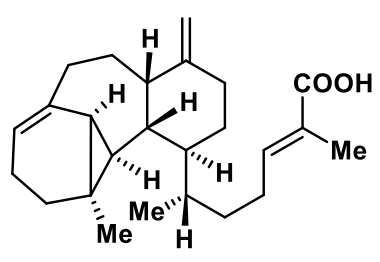

cerorubenic acid-III (1) (NOESY, $\mathrm{CD}_{2} \mathrm{Cl}_{2}, 500 \mathrm{MHz}$ )

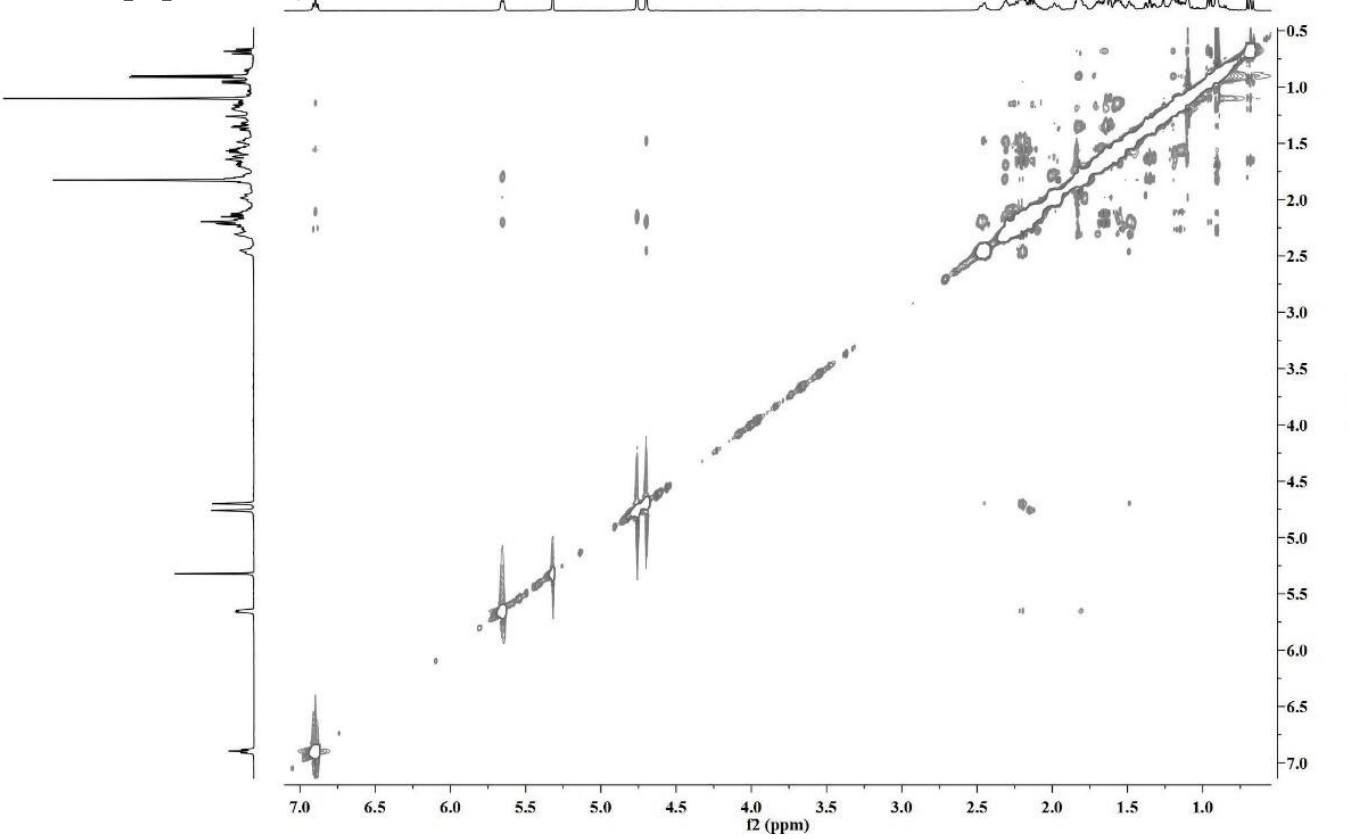

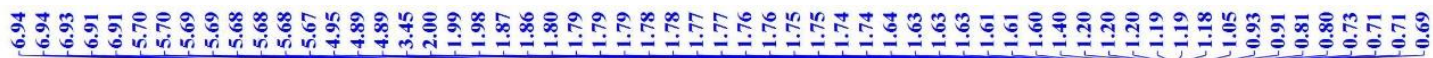

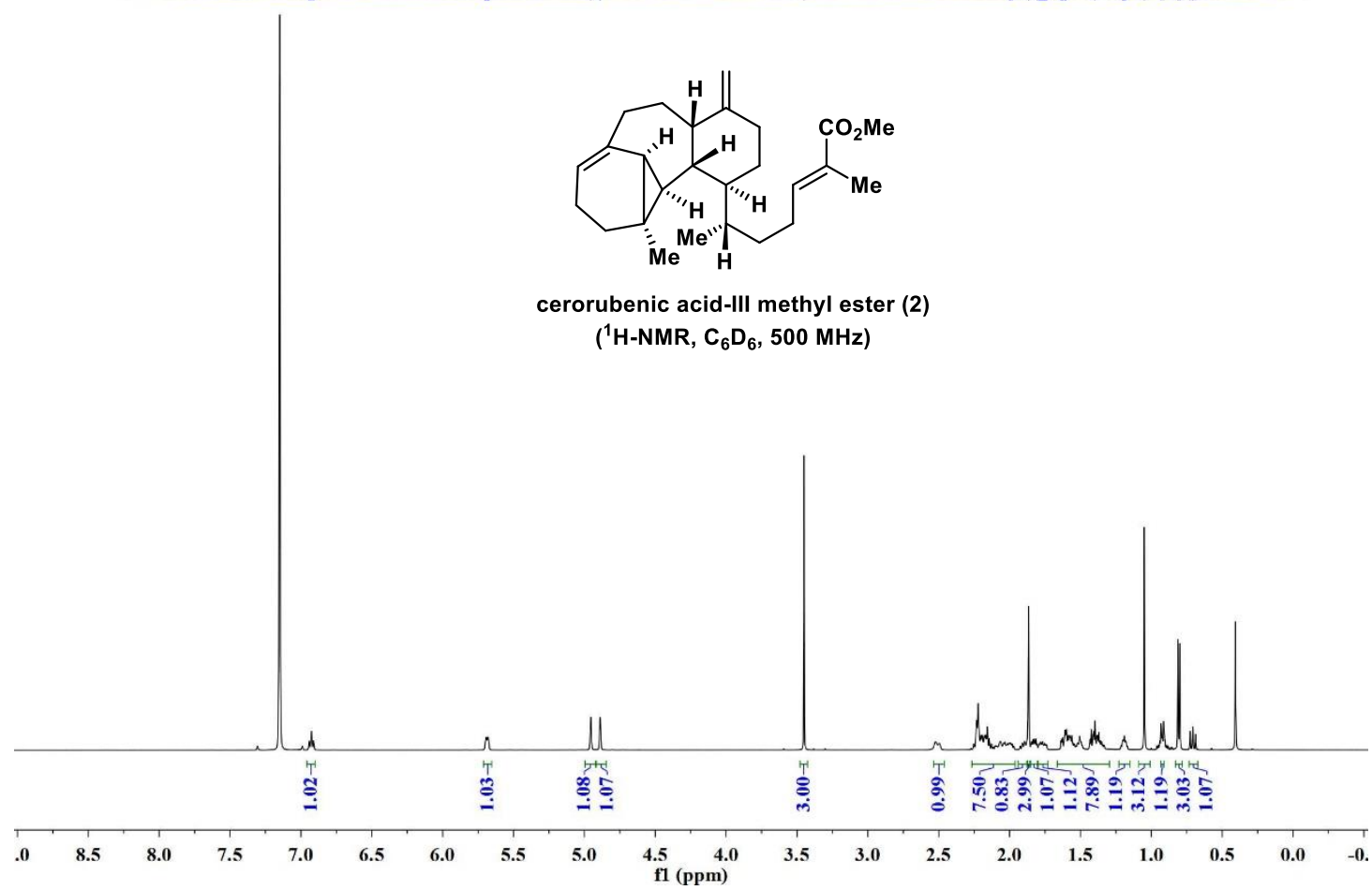




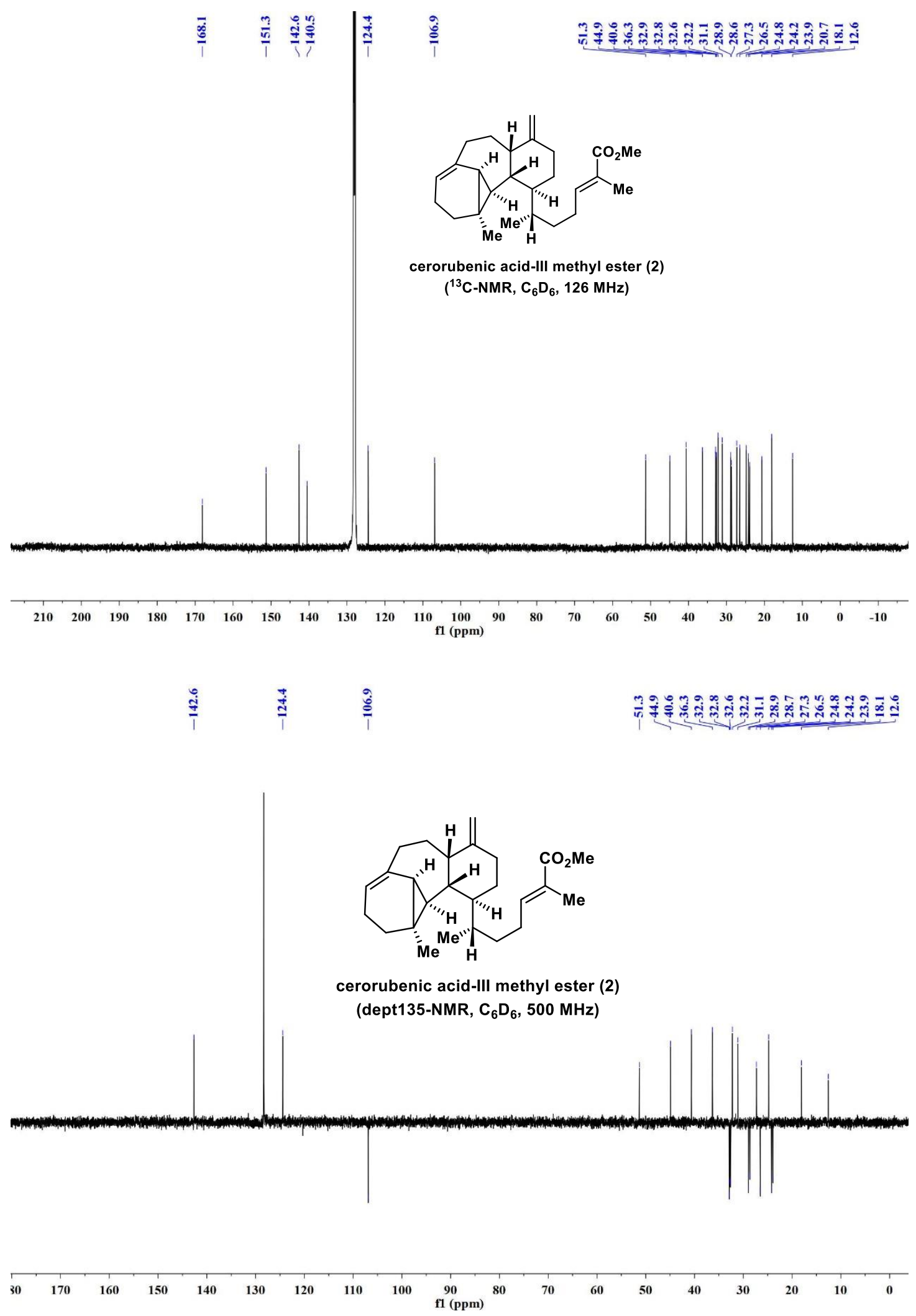



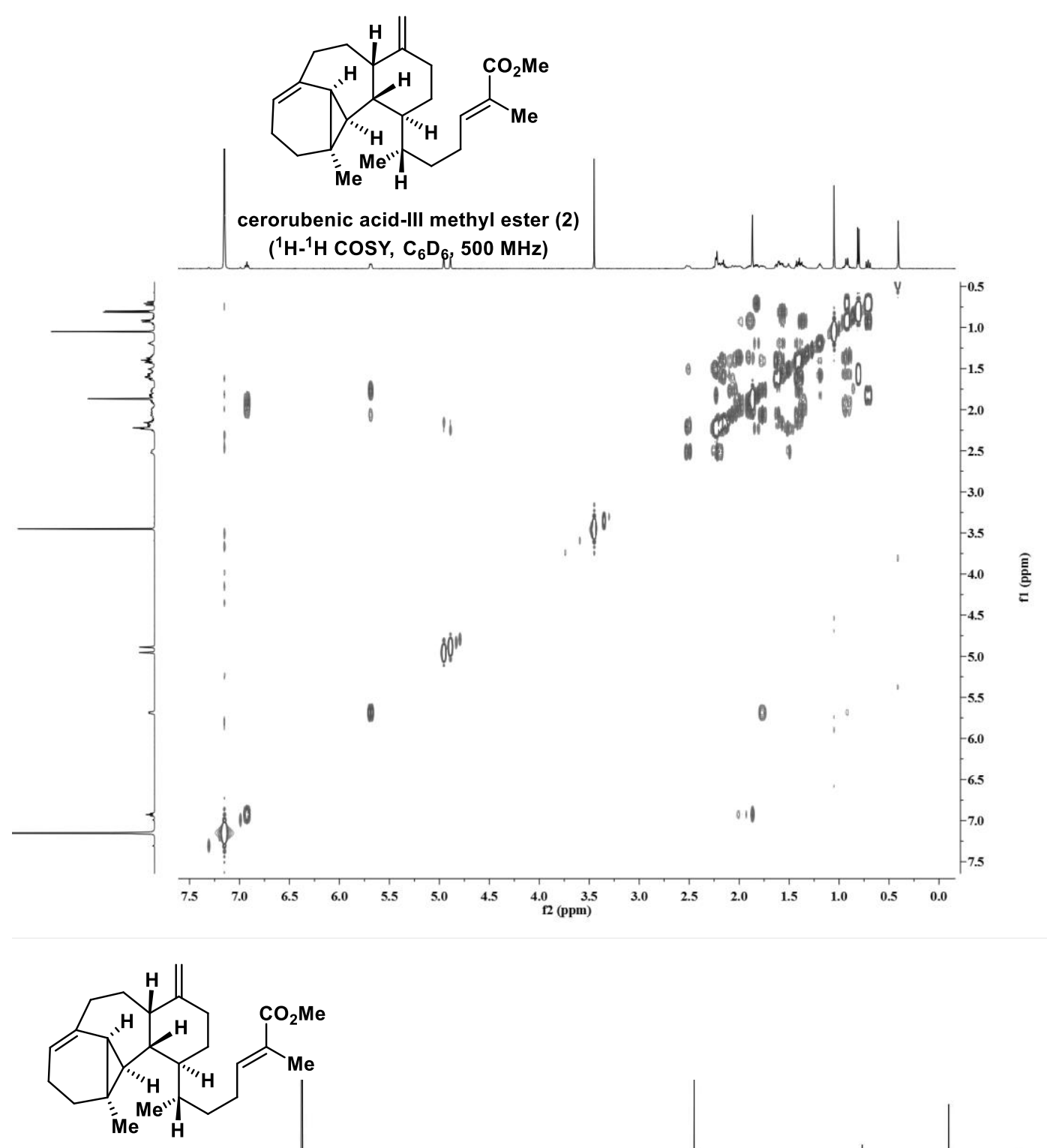

cerorubenic acid-III methyl ester (2)

(HMBC, $\mathrm{C}_{6} \mathrm{D}_{6}, 500 \mathrm{MHz}$ )

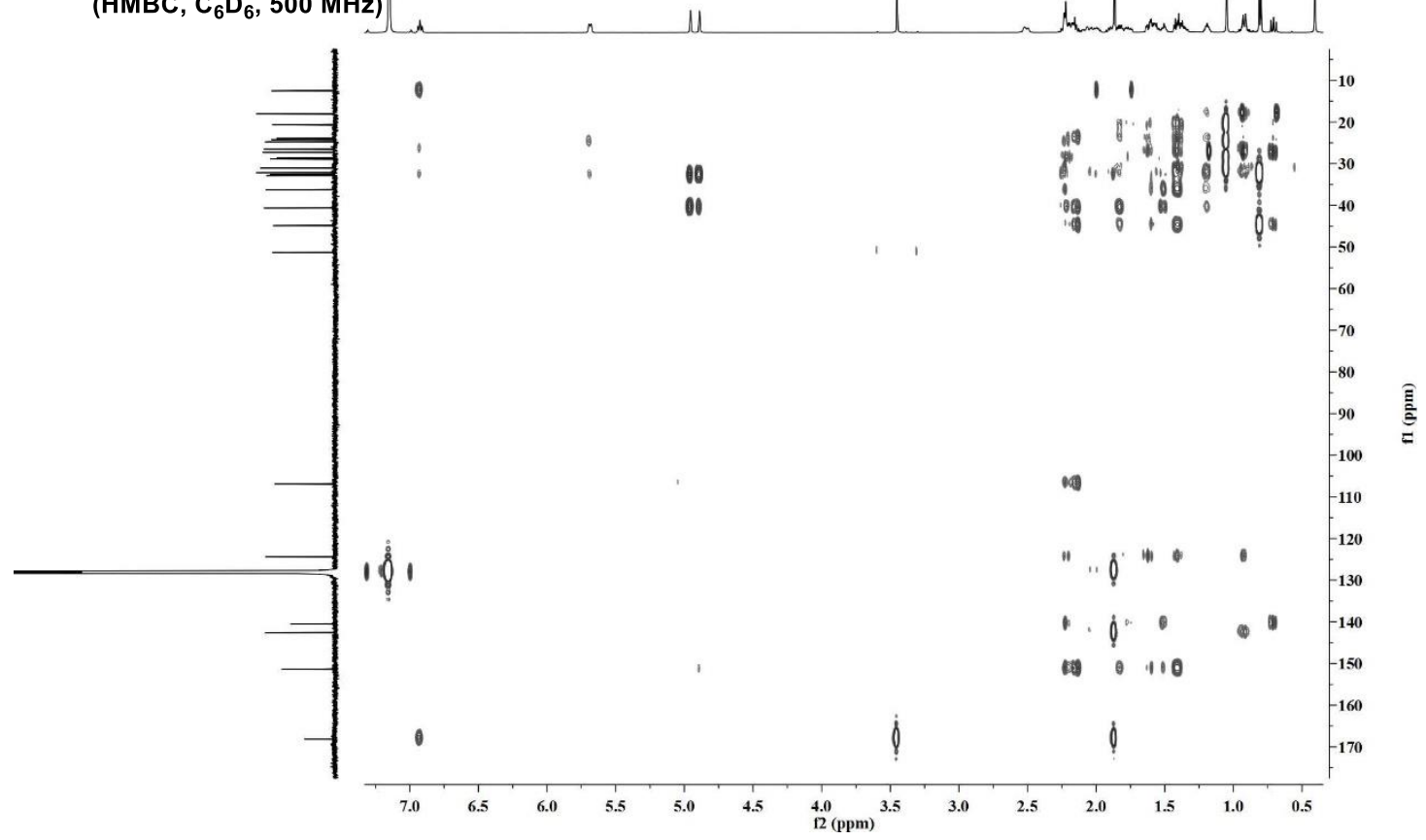




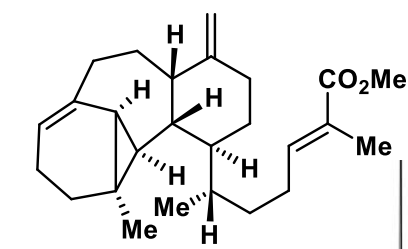

cerorubenic acid-III methyl ester (2)

(NOESY, $\mathrm{C}_{6} \mathrm{D}_{6}, 500 \mathrm{MHz}$ )

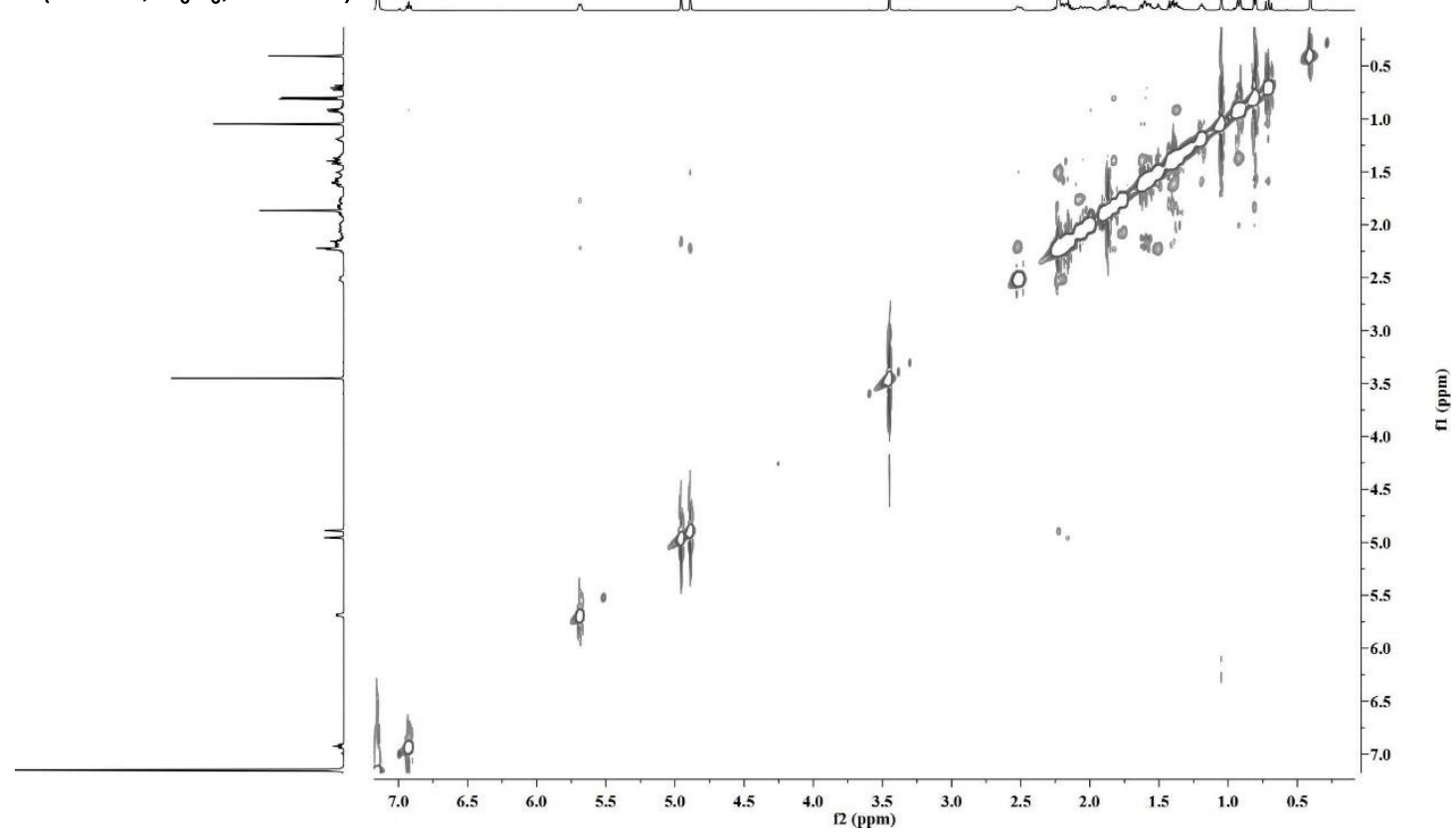

